\title{
A KOHNO-DRINFELD THEOREM FOR QUANTUM WEYL GROUPS
}

\author{
VALERIO TOLEDANO LAREDO
}

\begin{abstract}
Let $\mathfrak{g}$ be a complex, simple Lie algebra with Cartan subalgebra $\mathfrak{h}$ and Weyl group $W$. In MTL, we introduced a new, $W$-equivariant flat connection on $\mathfrak{h}$ with simple poles along the root hyperplanes and values in any finite-dimensional $\mathfrak{g}$-module $V$. It was conjectured in TL that its monodromy is equivalent to the quantum Weyl group action of the generalised braid group of type $\mathfrak{g}$ on $V$ obtained by regarding the latter as a module over the quantum group $U_{\hbar} \mathfrak{g}$. In this paper, we prove this conjecture for $\mathfrak{g}=\mathfrak{s l}_{n}$.
\end{abstract}

\section{INTRODUCTION}

One of the many virtues of quantum groups is their ability to describe the monodromy of certain first order systems of Fuchsian PDEs. If $U_{\hbar} \mathfrak{g}$ is the Drinfeld-Jimbo quantum group of the complex, simple Lie algebra $\mathfrak{g}$, the universal $R$-matrix of $U_{\hbar} \mathfrak{g}$ yields a representation of Artin's braid group on $n$ strings $B_{n}$ on the $n$-fold tensor product $V^{\otimes n}$ of

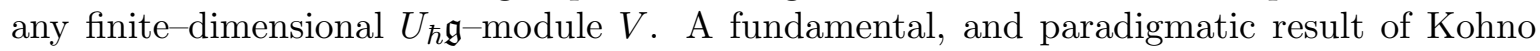
and Drinfeld establishes the equivalence of this representation with the monodromy of the Knizhnik-Zamolodchikov equations for $\mathfrak{g}$ with values in $V^{\otimes n}$ [Dr3, Dr4, Dr5, Ko1]. Lusztig, and independently Kirillov-Reshetikhin and Soibelman realised that $U_{\hbar \mathfrak{g}}$ also yields representations of another braid group, namely the generalised braid group $B_{\mathfrak{g}}$ of Lie type $\mathfrak{g}$ [Lu1, KR, So]. Whereas the $R$-matrix representation is a deformation of the natural action of the symmetric group $\mathfrak{S}_{n}$ on $n$-fold tensor products, these representations of $B_{\mathfrak{g}}$ deform the action of (a finite extension of) the Weyl group $W$ of $\mathfrak{g}$ on any finite-dimensional $\mathfrak{g}$-module $\mathrm{V}$.

The aim of this paper is to show that these quantum Weyl group representations describe the monodromy of the flat connection introduced in MTD and, independently, in [FMTV]. More precisely, realise $B_{\mathfrak{g}}$ as the fundamental group of the orbit space $\mathfrak{h}_{\text {reg }} / W$ of the set of regular elements of a Cartan subalgebra $\mathfrak{h}$ of $\mathfrak{g}$ under the action of $W[\mathrm{Br}$. Then, one can define a flat vector bundle $\left(\mathcal{V}, \nabla_{\kappa}\right)$ with fibre $V$ over $\mathfrak{h}_{\text {reg }} / W$ MTL. The connection $\nabla_{\kappa}$ depends upon a parameter $\hbar \in \mathbb{C}$ and it was conjectured in [TL] that, when $\hbar$ is regarded as a formal variable, its monodromy is equivalent to the quantum Weyl group action of $B_{\mathfrak{g}}$ on $V$. This conjecture was checked in [TL] for a number of pairs $(\mathfrak{g}, V)$ including vector representations of classical Lie algebras and adjoint representations of simple Lie algebras.

In the present paper, we prove this conjecture for $\mathfrak{g}=\mathfrak{s l}_{n}$, so that $B_{\mathfrak{g}}=B_{n}$. The proof relies on the Kohno-Drinfeld theorem for $U_{\hbar} \mathfrak{s l}_{k}$ via the use of the dual pair $\left(\mathfrak{g l}_{k}, \mathfrak{g l}_{n}\right)$. Our main observation is that the duality between $\mathfrak{g l}_{k}$ and $\mathfrak{g l}_{n}$ derived from their joint action on the space $\mathcal{M}_{k, n}$ of $k \times n$ matrices exchanges $\nabla_{\kappa}$ for $\mathfrak{s l}_{n}$ and the Knizhnik-Zamolodchikov connection for $\mathfrak{s l}_{k}$, thus acting as a simple-minded integral transform. This shows the equivalence of the monodromy representation of $\nabla_{\kappa}$ for $\mathfrak{s l}_{n}$ with a suitable $R$-matrix representation for $U_{\hbar} \mathfrak{s l}_{k}$. The proof is completed by noting that the duality between $U_{\hbar} \mathfrak{g l}_{k}$ and $U_{\hbar \mathfrak{g l}}$ exchanges the $R$-matrix representation of $U_{\hbar} \mathfrak{s l}_{k}$ with the quantum Weyl group representation of $U_{\hbar} \mathfrak{s l}_{n}$.

Date: 8 May 2001. 
This may be schematically summarised by the following diagram

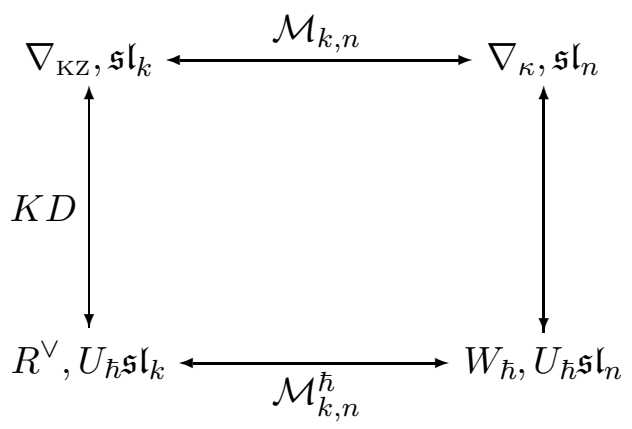

The structure of the paper is as follows. In section 2, we give the construction of the connection $\nabla_{\kappa}$ following [MTL. We show in section 3 that the duality between $\mathfrak{g l}_{k}$ and $\mathfrak{g l}_{n}$ identifies the Knizhnik-Zamolodchikov connection for $n$-fold tensor products of symmetric powers of the vector representation of $\mathfrak{s l}_{k}$ and the connection $\nabla_{\kappa}$ for $\mathfrak{s l}_{n}$. In section 1 we recall the definition of the Drinfeld-Jimbo quantum groups $U_{\hbar} \mathfrak{g l}_{k}$ and $U_{\hbar} \mathfrak{g l}_{n}$ and, in section 5 show how they jointly act on the quantum $k \times n$ matrix space $\mathcal{S}_{\hbar}\left(\mathcal{M}_{k, n}^{*}\right)$. The corresponding $R$-matrix and quantum Weyl group representations of $B_{n}$ on $\mathcal{S}_{\hbar}\left(\mathcal{M}_{k, n}^{*}\right)$ are shown to coincide in section 6. Section 7 contains our main result.

Acknowledgements. This paper was begun at the Reseach Institute for Mathematical Sciences of Kyoto University. I am very grateful to M. Kashiwara for his invitation to spend the summer of 1999 at RIMS and to RIMS for its hospitality and financial support. During my stay, I greatly benefitted from very stimulating and informative discussions with M. Kashiwara and B. Feigin. I also wish to express my gratitude to A. D'Agnolo, P. Baumann, B. Enriquez, J. Millson, R. Rouquier and P. Schapira for innumerable, useful and friendly conversations.

\section{Flat COnNECtions on $\mathfrak{h}_{\text {reg }}$}

The results in this section are due to J. Millson and the author [MTL. They were obtained independently by De Concini around 1995 (unpublished). Let $\mathfrak{g}$ be a complex, simple Lie algebra with Cartan subalgebra $\mathfrak{h}$ and root system $R \subset \mathfrak{h}^{*}$. Let $\mathfrak{h}_{\text {reg }}=\mathfrak{h} \backslash \bigcup_{\alpha \in R} \operatorname{Ker}(\alpha)$ be the set of regular elements in $\mathfrak{h}$ and $V$ a finite-dimensional $\mathfrak{g}$-module. We shall presently define a flat connection on the topologically trivial vector bundle $\mathfrak{h}_{\text {reg }} \times V$ over $\mathfrak{h}_{\text {reg. We }}$ need for this purpose the following simple flatness criterion due to Kohno [Ko2]. Let $B$ be a complex, finite-dimensional vector space and $\mathcal{A}=\left\{H_{i}\right\}_{i \in I}$ a finite collection of hyperplanes in $B$ determined by the linear forms $\phi_{i} \in B^{*}, i \in I$.

Lemma 2.1. Let $F$ be a finite-dimensional vector space and $\left\{r_{i}\right\} \subset \operatorname{End}(F)$ a family indexed by $I$. Then,

$$
\nabla=d-\sum_{i \in I} \frac{d \phi_{i}}{\phi_{i}} r_{i}
$$

defines a flat connection on $(B \backslash \mathcal{A}) \times F$ iff, for any subset $J \subseteq I$ maximal for the property that $\bigcap_{j \in J} H_{j}$ is of codimension 2, the following relations hold for any $j \in J$

$$
\left[r_{j}, \sum_{j^{\prime} \in J} r_{j^{\prime}}\right]=0
$$


For any $\alpha \in R$, choose root vectors $e_{\alpha} \in \mathfrak{g}_{\alpha}, f_{\alpha} \in \mathfrak{g}_{-\alpha}$ such that $\left[e_{\alpha}, f_{\alpha}\right]=h_{\alpha}=\alpha^{\vee}$ and let

$$
\kappa_{\alpha}=\frac{\langle\alpha, \alpha\rangle}{2}\left(e_{\alpha} f_{\alpha}+f_{\alpha} e_{\alpha}\right) \in U \mathfrak{g}
$$

be the truncated Casimir operator of the $\mathfrak{s l}_{2}(\mathbb{C})$-subalgebra of $\mathfrak{g}$ spanned by $e_{\alpha}, h_{\alpha}, f_{\alpha}$. Note that $\kappa_{\alpha}$ does not depend upon the particular choice of $e_{\alpha}$ and $f_{\alpha}$ and that $\kappa_{-\alpha}=\kappa_{\alpha}$.

Theorem 2.2. The one-form

$$
\nabla_{\kappa}^{h}=d-h \sum_{\alpha \succ 0} \frac{d \alpha}{\alpha} \kappa_{\alpha}=d-\frac{h}{2} \sum_{\alpha \in R} \frac{d \alpha}{\alpha} \kappa_{\alpha}
$$

defines, for any $h \in \mathbb{C}$, a flat connection on $\mathfrak{h}_{\mathrm{reg}} \times V$.

Proof. By lemma 2.1, we must prove that for any rank 2 subsystem $R_{0} \subseteq R$, the following holds for any $\alpha \in R_{0}^{+}=R_{0} \cap R^{+}$

$$
\left[\kappa_{\alpha}, \sum_{\beta \in R_{0}^{+}} \kappa_{\beta}\right]=0
$$

This may be proved by an explicit computation by considering in turn the cases where $R_{0}$ is of type $A_{1} \times A_{1}, A_{2}, B_{2}$ or $G_{2}$ but is more easily settled by the following elegant observation of A. Knutson [Kn]. Let $\mathfrak{g}_{0} \subseteq \mathfrak{g}$ be the semi-simple Lie algebra with root system $R_{0}, \mathfrak{h}_{0} \subset \mathfrak{h}$ its Cartan subalgebra and $C_{0} \in Z\left(U \mathfrak{g}_{0}\right)$ its Casimir operator. Then, $\sum_{\beta \in R_{0}^{+}} \kappa_{\beta}-C_{0}$ lies in $U \mathfrak{h}_{0}$ so that (2.5) holds since $\kappa_{\alpha}$ commutes with $\mathfrak{h}_{0}$

Let $G$ be the complex, connected and simply-connected Lie group with Lie algebra $\mathfrak{g}, T$ its torus with Lie algebra $\mathfrak{h}, N(T) \subset G$ the normaliser of $T$ and $W=N(T) / T$ the Weyl group of $G$. Let $B_{\mathfrak{g}}=\pi_{1}\left(\mathfrak{h}_{\mathrm{reg}} / W\right)$ be the generalised braid group of type $\mathfrak{g}$ and $\sigma: B_{\mathfrak{g}} \rightarrow N(T)$ a homomorphism compatible with

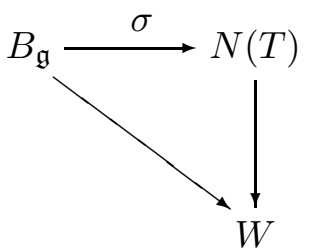

[Ti]. We regard $B_{\mathfrak{g}}$ as acting on $V$ via $\sigma$. Let $\widetilde{\mathfrak{h}_{\text {reg }}} \stackrel{p}{\rightarrow} \mathfrak{h}_{\text {reg }}$ be the universal cover of $\mathfrak{h}_{\text {reg }}$ and $\mathfrak{h}_{\text {reg }} / W$.

Proposition 2.3. The one-form $p^{*} \nabla_{\kappa}^{h}$ defines a $B_{\mathfrak{g}}$-equivariant flat connection on $\widetilde{\mathfrak{h}_{\text {reg }}} \times V=$ $p^{*}\left(\mathfrak{h}_{\mathrm{reg}} \times V\right)$. It therefore descends to a flat connection on the vector bundle

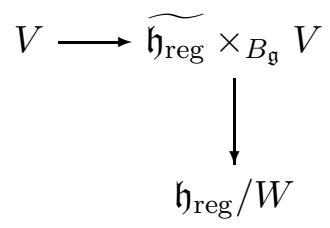

which is reducible with respect to the weight space decomposition of $V$ and unitary if $h \in i \mathbb{R}$.

Proof. The action of $B_{\mathfrak{g}}$ on $\Omega^{\bullet}\left(\widetilde{\mathfrak{h}_{\text {reg }}}, V\right)=\Omega^{\bullet}\left(\widetilde{\mathfrak{h}_{\text {reg }}}\right) \otimes V$ is given by $\gamma \rightarrow\left(\gamma^{-1}\right)^{*} \otimes \sigma(\gamma)$. Thus, if $\gamma \in B_{\mathfrak{g}}$ projects onto $w \in W$, we get using $p \cdot \gamma^{-1}=w^{-1} \cdot p$,

$$
\gamma p^{*} \nabla_{\kappa}^{h} \gamma^{-1}=d-\frac{h}{2} \sum_{\alpha \in R} d p^{*} w \alpha / p^{*} w \alpha \otimes \sigma(\gamma) \kappa_{\alpha} \sigma(\gamma)^{-1}
$$


Since $\kappa_{\alpha}=\frac{\langle\alpha, \alpha\rangle}{2}\left(e_{\alpha} f_{\alpha}+f_{\alpha} e_{\alpha}\right)$ is independent of the choice of the root vectors $e_{\alpha}, f_{\alpha}$, $\operatorname{Ad}(\sigma(\gamma)) \kappa_{\alpha}=\kappa_{w \alpha}$ and (2.8) is equal to $p^{*} \nabla_{\kappa}^{h}$ as claimed. $p^{*} \nabla_{\kappa}^{h}$ is flat by theorem 2.2, commutes with the fibrewise action of $\mathfrak{h}$ because each $\kappa_{\alpha}$ is of weight 0 and is unitary because the $\kappa_{\alpha}$ are self-adjoint

Thus, for any homomorphism $\sigma: B_{\mathfrak{g}} \rightarrow N(T)$ compatible with (2.6), proposition 2.3 yields a monodromy representation $\rho_{h}^{\sigma}: B_{\mathfrak{g}} \rightarrow G L(V)$ which permutes the weight spaces compatibly with $W$. By standard ODE theory, $\rho_{h}^{\sigma}$ depends analytically on the complex parameter $h$ and, when $h=0$, is equal to the action of $B_{\mathfrak{g}}$ on $V$ given by $\sigma$. We record for later use the following elementary

Proposition 2.4. Let $\gamma \in B_{\mathfrak{g}}=\pi_{1}\left(\mathfrak{h}_{\mathrm{reg}} / W\right)$ and $\widetilde{\gamma}:[0,1] \rightarrow \mathfrak{h}_{\text {reg }}$ be a lift of $\gamma$. Then,

$$
\rho_{h}^{\sigma}(\gamma)=\sigma(\gamma) \mathcal{P}(\widetilde{\gamma})
$$

where $\mathcal{P}(\widetilde{\gamma}) \in G L(V)$ is the parallel transport along $\widetilde{\gamma}$ for the connection $\nabla_{\kappa}^{h}$ on $\mathfrak{h}_{\text {reg }} \times V$.

PROOF. Let $\widetilde{\widetilde{\gamma}}:[0,1] \rightarrow \widetilde{\mathfrak{h}_{\text {reg }}}$ be a lift of $\gamma$ and $\widetilde{\gamma}$ so that $\widetilde{\gamma}(1)=\gamma^{-1} \widetilde{\widetilde{\gamma}}(0)$. Then, since the connection on $p^{*}\left(\mathfrak{h}_{\text {reg }} \times V\right)$ is the pull-back of $\nabla_{\kappa}^{h}$, and that on $\left(p^{*}\left(\mathfrak{h}_{\text {reg }} \times V\right)\right) / B_{\mathfrak{g}}$ the quotient of $p^{*} \nabla_{\kappa}^{h}$, we find

$$
\rho_{h}^{\sigma}(\gamma)=\mathcal{P}(\gamma)=\sigma(\gamma) \mathcal{P}(\widetilde{\widetilde{\gamma}})=\sigma(\gamma) \mathcal{P}(\widetilde{\gamma})
$$

By [B]], $B_{\mathfrak{g}}$ is presented on generators $T_{i}, i=1 \ldots n$ labelled by a choice of simple roots $\alpha_{i}$ of $R$ with relations

$$
T_{i} T_{j} T_{i} \cdots=T_{j} T_{i} T_{j} \cdots
$$

for any $1 \leq i<j \leq n$ where each side of (2.11) has a number of factors equal to the order of $s_{i} s_{j}$ in $W$ and $s_{k} \in W$ is the orthogonal reflection across the hyperplane $\operatorname{Ker}\left(\alpha_{k}\right)$. $T_{i}$ projects onto $s_{i} \in W$. An explicit choice of representatives of $T_{1}, \ldots, T_{n}$ in $\pi_{1}\left(\mathfrak{h}_{\mathrm{reg}} / W\right)$ may be given as follows. Let $t \in \mathfrak{h}_{\text {reg }}$ lie in the fundamental Weyl chamber so that $\langle t, \alpha\rangle>0$ for any $\alpha \in R_{+}$. Note that for any simple root $\alpha_{i}$, the intersection $t_{\alpha_{i}}=t-\frac{1}{2}\left\langle t, \alpha_{i}\right\rangle \alpha_{i}^{\vee}$ of the affine line $t+\mathbb{C} \cdot \alpha_{i}^{\vee}$ with $\operatorname{Ker}\left(\alpha_{i}\right)$ does not lie in any other root hyperplane $\operatorname{Ker}(\beta), \beta \in R \backslash\left\{\alpha_{i}\right\}$. Indeed, if $\left\langle t_{\alpha_{i}}, \beta\right\rangle=0$ then

$$
2\langle t, \beta\rangle=\left\langle t, \alpha_{i}\right\rangle\left\langle\alpha_{i}^{\vee}, \beta\right\rangle=\left\langle t, \beta-s_{i} \beta\right\rangle
$$

whence $\langle t, \beta\rangle=-\left\langle t, s_{i} \beta\right\rangle$, a contradiction since $s_{i}$ permutes positive roots different from $\alpha_{i}$. Let now $D$ be an open disc in $t+\mathbb{C} \cdot \alpha_{i}^{\vee}$ of center $t_{\alpha_{i}}$ such that its closure $\bar{D}$ does not intersect any root hyperplane other than $\operatorname{Ker}\left(\alpha_{i}\right)$. Consider the path $\gamma_{i}:[0,1] \rightarrow t+\mathbb{C} \cdot \alpha_{i}^{\vee}$ from $t$ to $s_{i} t$ determined by $\left.\gamma_{i}\right|_{[0,1 / 3] \cup[2 / 3,1]}$ is affine and lies in $t+\mathbb{R} \cdot \alpha^{\vee} \backslash D, \gamma_{i}(1 / 3), \gamma_{i}(2 / 3) \in \partial \bar{D}$ and $\left.\gamma_{i}\right|_{[1 / 3,2 / 3]}$ is a semicircular arc in $\partial \bar{D}$, positively oriented with respect to the natural orientation of $t+\mathbb{C} \cdot \alpha_{i}$. Then, the image of $\gamma_{i}$ in $\mathfrak{h}_{\mathrm{reg}} / W$ is a representative of $T_{i}$ in $\pi_{1}\left(\mathfrak{h}_{\mathrm{reg}} / W, W t\right)$ Br].

\section{KNIZHNIK-ZAMOLODCHIKOV EQUATIONS AND DUAL PAIRS}

We show in this section that the joint action of $\mathfrak{g l}_{k}$ and $\mathfrak{g l}_{n}$ on the space $\mathcal{M}_{k, n}$ of $k \times n$ matrices identifies the connection $\nabla_{\kappa}$ for $\mathfrak{g}=\mathfrak{s l}_{n}$ and the Knizhnik-Zamolodchikov connection for $\mathfrak{s l}_{k}$. 
Let $\mathcal{S}\left(\mathcal{M}_{k, n}^{*}\right)=\mathbb{C}\left[x_{11}, \ldots, x_{k n}\right]$ be the algebra of polynomial functions on $\mathcal{M}_{k, n}$. The group $G L_{k} \times G L_{n}$ acts on $\mathcal{S}\left(\mathcal{M}_{k, n}^{*}\right)$ by

$$
\left(g_{k}, g_{n}\right) p(x)=p\left(g_{k}^{t} x g_{n}\right)
$$

and leaves the homogeneous components $\mathcal{S}^{d}\left(\mathcal{M}_{k, n}^{*}\right)$ of $\mathcal{S}\left(\mathcal{M}_{k, n}^{*}\right), d \in \mathbb{N}$, invariant. The decomposition of $\mathcal{S}\left(\mathcal{M}_{k, n}^{*}\right)$ under $G L_{k} \times G L_{n}$ is well-known (see e.g., [Zh, §132] which we follow closely or [Mc, §1.4] Đ). Let $N_{k}, N_{n}$ be the groups of $k \times k$ and $n \times n$ upper triangular unipotent matrices respectively.

\section{Lemma 3.1.}

$$
\mathcal{S}\left(\mathcal{M}_{k, n}^{*}\right)^{N_{k} \times N_{n}}=\mathbb{C}\left[\Delta_{1}, \ldots, \Delta_{\min (k, n)}\right]
$$

where $\Delta_{l}(x)=\operatorname{det}\left(x_{i j}\right)_{1 \leq i, j \leq l}$ is the lth principal minor of the matrix $x$.

ProOf. Assume for simplicity that $k \leq n$. Let $\mathcal{D} \subset \mathcal{S}\left(\mathcal{M}_{k, n}^{*}\right)$ be the subset of matrices $x$ such that $\Delta_{i}(x) \neq 0$ for $i=1 \ldots k$. By the Gauss decomposition, any $x \in \mathcal{D}$ is conjugate under $N_{k}^{t} \times N_{n}$ to a unique $k \times n$ matrix $d(x)$ with the same principal minors as $x$, diagonal principal $k \times k$ block and the remaining columns equal to zero. Consider now the $k \times n$ matrix

$$
m(x)=\left(\begin{array}{ccccccccc}
\Delta_{1}(x) & \Delta_{2}(x) & \Delta_{3}(x) & \cdots & \Delta_{k-1}(x) & \Delta_{k}(x) & 0 & \cdots & 0 \\
-1 & 0 & 0 & \cdots & 0 & 0 & 0 & \cdots & 0 \\
0 & -1 & 0 & \cdots & 0 & 0 & 0 & \cdots & 0 \\
\vdots & \vdots & \vdots & \cdots & \vdots & \vdots & \vdots & \cdots & \vdots \\
0 & 0 & 0 & \cdots & -1 & 0 & 0 & \cdots & 0
\end{array}\right)
$$

Since $\Delta_{i}(m(x))=\Delta_{i}(x), 1 \leq i \leq k, m(x)$ is also conjugate to $d(x)$, and therefore to $x$, under $N_{k}^{t} \times N_{n}$. Thus, by density of $\mathcal{D}$, a polyonomial $p \in \mathcal{S}\left(\mathcal{M}_{k, n}^{*}\right)$ is invariant under $N_{k} \times N_{n}$ iff it is a function of $m(x)$ and therefore iff it is a polynomial in $\Delta_{1}, \ldots, \Delta_{k}$

Let $\mathbb{Y}_{p} \subset \mathbb{N}^{p}$ be the set of Young diagrams with at most $p$ rows. For $\lambda \in \mathbb{Y}_{p}$, set $|\lambda|=\sum_{i=1}^{p} \lambda_{i}$ and let $V_{\lambda}^{(p)}$ be the irreducible representation of $G L_{p}(\mathbb{C})$ of highest weight $\lambda$.

Theorem 3.2. As $G L_{k} \times G L_{n}-$ modules,

$$
\mathcal{S}^{d}\left(\mathcal{M}_{k, n}^{*}\right) \cong \bigoplus_{\substack{\lambda \in \mathbb{Y}_{\min (k, n)}, \lambda \\|\lambda|=d}} V_{\lambda}^{(k)} \otimes V_{\lambda}^{(n)}
$$

Proof. Assume again $k \leq n$ for simplicity. By lemma 3.1, the highest weight vectors for the action of $G L_{k}(\mathbb{C}) \times G L_{n}(\mathbb{C})$ on $\mathcal{S}\left(\mathcal{M}_{k, n}^{*}\right)$ are the polynomials in $\Delta_{1}, \ldots, \Delta_{k}$ which are eigenvectors for the torus of $G L_{k} \times G L_{n}$. Since $\Delta_{l}$ is of weight $\varpi_{l}^{(k)} \oplus \varpi_{l}^{(n)}$, where $\varpi_{l}^{(p)}$ is the lth fundamental weight of $G L_{p}$, the highest weight vectors are the monomials $\Delta_{1}^{m_{1}} \cdots \Delta_{k}^{m_{k}}$ with corresponding pair of Young diagrams $(\lambda, \lambda)$ where

$$
\lambda=\left(m_{1}+\cdots+m_{k}, m_{2}+\cdots+m_{k}, \ldots, m_{k}\right)
$$

Thus, (3.4) holds since $\Delta_{l}$ is a homogeneous function of degree $l$

As a $\mathfrak{g l}_{k}$-module,

$$
\mathcal{S}\left(\mathcal{M}_{k, n}^{*}\right)=\mathbb{C}\left[x_{11}, \ldots, x_{k 1}\right] \otimes \cdots \otimes \mathbb{C}\left[x_{1 n}, \ldots, x_{k n}\right]
$$

\footnotetext{
${ }^{1}$ I am grateful to M. Vergne for pointing out that the decomposition (3.4) was known long before [Zh] and the work of Howe $\mathrm{Ho}$ and to M. Brion for providing the reference $\mathrm{Mc}$.
} 
and is therefore acted upon by the $\mathfrak{g l}_{k}$-intertwiners $\widetilde{\Omega}_{i j}^{(k)}, 1 \leq i<j \leq n$, defined by

$$
\widetilde{\Omega}_{i j}^{(k)}=\sum_{a} 1^{\otimes(i-1)} \otimes X_{a} \otimes 1^{\otimes(j-i-1)} \otimes X^{a} \otimes 1^{\otimes(n-j)}
$$

where $\left\{X_{a}\right\},\left\{X^{a}\right\}$ are dual basis of $\mathfrak{g l}_{k}$ with respect to the pairing $\langle X, Y\rangle=\operatorname{tr}(X Y)$. On the other hand, as a $\mathfrak{g l}_{n}$-module, $\mathcal{S}\left(\mathcal{M}_{k, n}^{*}\right)$ is acted upon by the operators $\kappa_{i j}^{(n)}, 1 \leq i<j \leq n$, where

$$
\kappa_{i j}^{(n)}=e_{\alpha} f_{\alpha}+f_{\alpha} e_{\alpha}
$$

is the truncated Casimir operator of the $\mathfrak{s l}_{2}(\mathbb{C})$-subalgebra of $\mathfrak{g l}_{n}$ corresponding to the root $\alpha=\theta_{i}-\theta_{j}$. Let $e_{1}, \ldots, e_{p}$ be the canonical basis of $\mathbb{C}^{p}$ and $E_{a b}^{(p)} e_{c}=\delta_{b c} e_{a}, 1 \leq a, b \leq p$ the corresponding basis of $\mathfrak{g l}_{p}$ with dual basis $E_{b a}^{(p)}$. Let $1 \leq i<j \leq n$, then

Proposition 3.3. The following holds on $\mathcal{S}\left(\mathcal{M}_{k, n}^{*}\right)$

$$
2 \widetilde{\Omega}_{i j}^{(k)}=\kappa_{i j}^{(n)}-E_{i i}^{(n)}-E_{j j}^{(n)}
$$

Proof. By (3.7), $\widetilde{\Omega}_{i j}^{(k)}$ acts on $\mathcal{S}\left(\mathcal{M}_{k, n}^{*}\right)$ as

$$
\widetilde{\Omega}_{i j}^{(k)}=\sum_{1 \leq a, b \leq k} x_{a i} \partial_{b i} x_{b j} \partial_{a j}
$$

where $x_{r c}$ and $\partial_{r c}$ are the operators of multiplication by and derivation with respect to $x_{r c}$. On the other hand, given that the $\mathfrak{s l}_{2}(\mathbb{C})$-triple $\left\{e_{\alpha}, h_{\alpha}, f_{\alpha}\right\}$ corresponding to the root $\alpha=\theta_{i}-\theta_{j}$ of $\mathfrak{g l}_{n}$ is $\left\{E_{i j}^{(n)}, E_{i i}^{(n)}-E_{j j}^{(n)}, E_{j i}^{(n)}\right\}$, the following holds on $\mathcal{S}\left(\mathcal{M}_{k, n}^{*}\right)$

$$
\kappa_{i j}^{(n)}=\sum_{1 \leq a, b \leq k} x_{a i} \partial_{a j} x_{b j} \partial_{b i}+x_{b j} \partial_{b i} x_{a i} \partial_{a j}
$$

Substracting, we find

$$
2 \widetilde{\Omega}_{i j}^{(k)}-\kappa_{i j}^{(n)}=-\sum_{1 \leq a, b \leq k} \delta_{a b} x_{a i} \partial_{b i}+\delta_{a b} x_{b j} \partial_{a j}=-E_{i i}^{(n)}-E_{j j}^{(n)}
$$

as claimed

Let $\lambda \in \mathbb{Y}_{\min (k, n)}$ and $V_{\lambda}^{(n)}$ the corresponding simple $G L_{n}-$ module. By theorem 3.2, $V_{\lambda}^{(n)}$ may be identified with the subspace of vectors of highest weight $\lambda$ for the action of $\mathfrak{g l}_{k}$ on $\mathcal{S}\left(\mathcal{M}_{k, n}^{*}\right)$. Denote by $\iota: V_{\lambda} \rightarrow \mathcal{S}\left(\mathcal{M}_{k, n}^{*}\right)$ the corresponding $\mathfrak{g l}_{n}$-equivariant embedding and let $\mu=\left(\mu_{1}, \ldots, \mu_{n}\right) \in \mathbb{N}^{n}$ be a weight of $V_{\lambda}^{(n)}$.

Lemma 3.4. The embedding ८ maps the subspace $V_{\lambda}^{(n)}[\mu] \subset V_{\lambda}^{(n)}$ of weight $\mu$ onto the subspace $M_{\lambda}^{\mu}$ of vectors of highest weight $\lambda$ for the action of $\mathfrak{g l}_{k}$ on

$$
S^{\mu} \mathbb{C}^{k}=S^{\mu_{1}} \mathbb{C}^{k} \otimes \cdots \otimes S^{\mu_{n}} \mathbb{C}^{k} \subset \mathbb{C}\left[x_{11}, \ldots, x_{k 1}\right] \otimes \cdots \otimes \mathbb{C}\left[x_{1 n}, \ldots, x_{k n}\right]
$$

where $S^{\mu_{j}} \mathbb{C}^{k}$ is the space of polynomials in $x_{1 j}, \ldots, x_{k j}$ which are homogeneous of degree $\mu_{j}$. The corresponding isomorphism

$$
\bigoplus_{\nu \in \mathfrak{S}_{n} \mu} V_{\lambda}^{(n)}[\nu] \cong \bigoplus_{\nu \in \mathfrak{S}_{n} \mu} M_{\lambda}^{\nu}
$$

is equivariant with respect to $\mathfrak{S}_{n}$ which acts on $\bigoplus_{\nu \in \mathfrak{S}_{n} \mu} S^{\nu} \mathbb{C}^{k}$ by permuting the tensor factors and on $V_{\lambda}$ by regarding $\mathfrak{S}_{n}$ as the subgroup of permutation matrices of $G L_{n}(\mathbb{C})$. 
Proof. The equality $\iota\left(V_{\lambda}^{(n)}[\mu]\right)=M_{\lambda}^{\mu}$ holds because $S^{\mu} \mathbb{C}^{k}$ is the subspace of $\mathcal{S}\left(\mathcal{M}_{k, n}^{*}\right)$ of weight $\mu$ for the $\mathfrak{g l}_{n}$-action since $E_{i i}^{(n)} x_{r j}^{m}=\delta_{i j} m x_{r j}^{m}$. The $\mathfrak{S}_{n}$-equivariance stems from the fact that the permutation of the tensor factors in $S^{\bullet} \mathbb{C}^{k} \otimes \cdots \otimes S^{\bullet} \mathbb{C}^{k} \cong S^{\bullet}\left(\mathbb{C}^{k} \otimes \mathbb{C}^{n}\right)$ is given by the action of $\mathfrak{S}_{n} \subset G L_{n}(\mathbb{C})$ action on $\mathbb{C}^{n}$

Let $\mathcal{D}_{n}=\left\{\left(z_{1}, \ldots, z_{n}\right) \in \mathbb{C}^{n} \mid z_{i}=z_{j}\right.$ for some $\left.1 \leq i<j \leq n\right\}$ and $X_{n}=\mathbb{C}^{n} \backslash \mathcal{D}_{n}$. Regard $\mathbb{C}_{0}^{n}=\left\{\left(z_{1}, \ldots, z_{n}\right) \in \mathbb{C}^{n} \mid \sum_{j=1}^{n} z_{j}=0\right\}$ as the Cartan subalgebra of diagonal matrices in $\mathfrak{s l}_{n}$ and $X_{n}^{0}=\mathbb{C}_{0}^{n} \backslash \mathcal{D}_{n}$ as the set of its regular elements. Since the inclusion $X_{n}^{0} \subset X_{n}$ is a homotopy equivalence, $\pi_{1}\left(X_{n}\right) \cong \pi_{1}\left(X_{n}^{0}\right)=B_{n}$ are generated by $T_{1} \ldots T_{n-1}$ with

$$
\begin{aligned}
T_{i} T_{j} & =T_{j} T_{i} & & \text { if }|i-j| \geq 2 \\
T_{i} T_{i+1} T_{i} & =T_{i+1} T_{i} T_{i+1} & & i=1 \ldots n-1
\end{aligned}
$$

Define $\Omega_{i j}^{(k)} \in \operatorname{End}_{\mathfrak{g r}_{k}}\left(S^{\mu} \mathbb{C}^{k}\right)$ by (3.7) where now $\left\{X_{a}\right\},\left\{X^{a}\right\}$ are dual basis of $\mathfrak{s l}_{k}$ and extend the connection (2.4) to $X_{n}$ in the obvious way. The following is the main result of this section.

Theorem 3.5. $f: X_{n} \rightarrow M_{\lambda}^{\mu} \subset S^{\mu_{1}} \mathbb{C}^{k} \otimes \cdots \otimes S^{\mu_{n}} \mathbb{C}^{k}$ is a horizontal section of the KnizhnikZamolodchikov connection

$$
\nabla_{\mathrm{KZ}}^{\bar{h}}=d-\bar{h} \sum_{1 \leq i<j \leq n} \frac{d z_{i}-d z_{j}}{z_{i}-z_{j}} \Omega_{i j}^{(k)}
$$

iff the $V_{\lambda}^{(n)}[\mu]$-valued function $g=f \cdot \prod_{1 \leq i<j \leq n}\left(z_{i}-z_{j}\right)^{h\left(\mu_{i}+\mu_{j}+2 \mu_{i} \mu_{j} / k\right)}$ is a horizontal section of

$$
\nabla_{\kappa}^{h}=d-h \sum_{1 \leq i<j \leq n} \frac{d z_{i}-d z_{j}}{z_{i}-z_{j}} \kappa_{i j}^{(n)}
$$

where $\bar{h}=2 h$.

PROOF. Let $1^{(k)}=\sum_{i=1}^{k} E_{i i}^{(k)}$ be the generator of the centre of $\mathfrak{g l}_{k}$ so that, in obvious notation, $\widetilde{\Omega}_{i j}^{(k)}=\Omega_{i j}^{(k)}+\frac{1}{k} \pi_{i}\left(1^{(k)}\right) \pi_{j}\left(1^{(k)}\right)$. The operators $2 \widetilde{\Omega}_{i j}^{(k)}$ and $\kappa_{i j}^{(n)}$ both act on $M_{\lambda}^{\mu} \cong V_{\lambda}^{(n)}[\mu]$ and, by proposition 3.3, their restrictions differ by $-\mu_{i}-\mu_{j}$. The claim follows since, for any $1 \leq l \leq n, \pi_{l}\left(1^{(k)}\right)$ acts on $S^{\mu} \mathbb{C}^{k}$ as multiplication by $\mu_{l}$

REMARK. When $k=2$ and $\lambda$ is of the form $(|\mu| / 2,|\mu| / 2,0, \ldots, 0)$, where $|\mu|=\sum_{i=1} \mu_{i}$, theorem 3.5 is a representation-theoretic analogue of the coincidence between the KapovichMillson bending flows on the space of $n$-gons in $\mathbb{R}^{3}$ with side lengths $\mu_{1}, \ldots, \mu_{n}$ [KM] and the Gel'fand-Cetlin flows on the Grassmannian $\mathrm{Gr}_{2}\left(\mathbb{C}^{n}\right)$ GS observed by Hausmann and Knutson in the context of Gel'fand-McPherson duality [HK]. I am grateful to J. Millson for a careful explanation of this coincidence.

REMARK. An interesting relation between $\nabla_{\kappa}$ and the Knizhnik-Zamolodchikov connection was recently noted by Felder, Markov, Tarasov and Varchenko in [FMTV], where a variant of the connection (2.4) is independently introduced and studied. One of the main results of [FMTV] is that, for any simple Lie algebra $\mathfrak{g}$, the connection $\nabla_{\kappa}$ with values in a tensor product $V_{1} \otimes \cdots \otimes V_{n}$ of $n$ simple $\mathfrak{g}$-modules is, when supplemented by suitable dynamical parameters, bispectral to (i.e., commutes with) the Knizhnik-Zamolodchikov connection for $\mathfrak{g}$ with values in the same $n$-fold tensor product. An analogous result is obtained in [TV] for a difference analogue of the connection $\nabla_{\kappa}$. By comparison, theorem 3.5 can only hold for $\mathfrak{g}=\mathfrak{s l}_{n}$, since it relies on the 'coincidence' of the regular Cartan of $\mathfrak{g l}_{n}$ with the configuration 
space of $n$ ordered points in $\mathbb{C}$, and asserts the equality of the two connections.

To relate the monodromy representations of $B_{n}$ corresponding to $\nabla_{\mathrm{KZ}}^{\bar{h}}$ and $\nabla_{\kappa}^{h}$, we need to specify how these induce flat connections on $X_{n} / \mathfrak{S}_{n}$ and $X_{n}^{0} / \mathfrak{S}_{n}$ respectively. For $\nabla_{\mathrm{KZ}}^{\bar{h}}$, we let $\mathfrak{S}_{n}$ act on the fibre

$$
\bigoplus_{\nu \in \mathfrak{S}_{n} \mu} M_{\lambda}^{\nu} \subset \bigoplus_{\nu \in \mathfrak{S}_{n} \mu} \mathcal{S}^{\nu} \mathbb{C}^{k}
$$

by permuting the tensor factors and take the quotient connection. For $\nabla_{\kappa}^{h}$, we use the construction of proposition 2.3 and the homomorphism $\sigma: B_{n} \rightarrow S L_{n}(\mathbb{C})$ given by

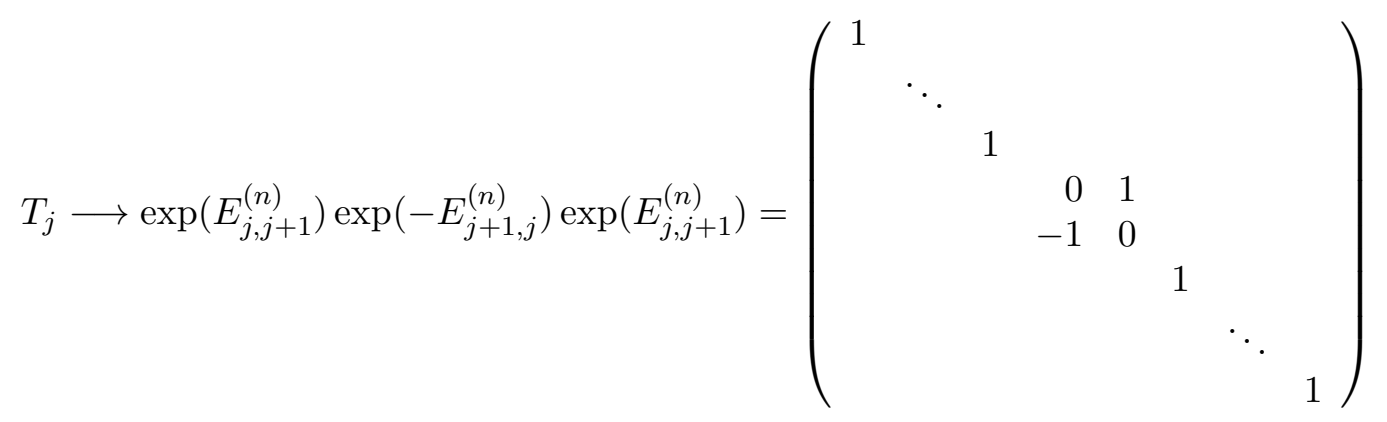

where the off-diagonal terms are the $(j, j+1)$ and $(j+1, j)$ entries. A direct computation, or [Ti, thm. 3.3], show that the assignement (3.20) does indeed extend to a homomorphism $B_{n} \rightarrow S L_{n}(\mathbb{C})$. Choose the generators $T_{1} \ldots T_{n-1}$ of $B_{n}$ as at the end of section 2 .

Corollary 3.6. Let $\mu$ be a weight of $V_{\lambda}$ and

$$
\pi_{\kappa}^{h}: B_{n} \rightarrow G L\left(\bigoplus_{\nu \in \mathfrak{S}_{n} \mu} V_{\lambda}[\nu]\right), \quad \pi_{\mathrm{KZ}}^{\bar{h}}: B_{n} \rightarrow G L\left(\bigoplus_{\nu \in \mathfrak{S}_{n} \mu} M_{\lambda}^{\nu}\right)
$$

the monodromy representations of the braid group $B_{n}$ corresponding to the connections (3.18) and (3.17) respectively. Then, for any $j=1 \ldots n-1$,

$$
\pi_{\mathrm{KZ}}^{2 h}\left(T_{j}\right)=\pi_{\kappa}^{h}\left(T_{j}\right) \cdot e^{-\pi i h\left(E_{j j}^{(n)}+E_{j+1 j+1}^{(n)}+2 E_{j j}^{(n)} E_{j+1 j+1}^{(n)} / k\right)} \cdot e^{i \pi E_{j j}^{(n)}}
$$

Proof. Let $s_{j} \in S L_{n}(\mathbb{C})$ be the right-hand side of $(3.20)$ so that $s_{j}=(j j+1) \cdot e^{i \pi E_{j j}^{(n)}}$ in $G L_{n}(\mathbb{C})$. Let $\mathcal{P}_{\mathrm{KZ}}^{\bar{h}}, \mathcal{P}_{\kappa}^{h}$ denote parallel transport for $\nabla_{\mathrm{KZ}}^{\bar{h}}$ and $\nabla_{\kappa}^{h}$ respectively. Then, by theorem 3.5 and proposition 2.4, the following holds on $M_{\lambda}^{\nu} \cong V_{\lambda}[\nu]$,

$$
\begin{aligned}
\pi_{\mathrm{KZ}}^{2 h}\left(T_{j}\right) & =(j j+1) \mathcal{P}_{\mathrm{KZ}}^{2 h}\left(T_{j}\right) \\
& =(j j+1) e^{-\pi i h\left(\nu_{j}+\nu_{j+1}+2 \nu_{j} \nu_{j+1} / k\right)} \mathcal{P}_{\kappa}^{h}\left(T_{j}\right) \\
& =s_{j} \mathcal{P}_{\kappa}^{h}\left(T_{j}\right) e^{i \pi E_{j j}^{(n)}} e^{-\pi i h\left(E_{j j}^{(n)}+E_{j+1 j+1}^{(n)}+2 E_{j j}^{(n)} E_{j+1 j+1}^{(n)} / k\right)} \\
& =\pi_{\kappa}^{h}\left(T_{j}\right) e^{-\pi i h\left(E_{j j}^{(n)}+E_{j+1 j+1}^{(n)}+2 E_{j j}^{(n)} E_{j+1 j+1}^{(n)} / k\right)} e^{i \pi E_{j j}^{(n)}}
\end{aligned}
$$

as claimed

\section{The QUANTUM GrouP $U_{\hbar} \mathfrak{g l}_{p}$}

In this, and the following sections, we work over the ring $\mathbb{C} \llbracket \hbar \rrbracket$ of formal power series in the variable $\hbar$. All tensor products of $\mathbb{C} \llbracket \hbar \rrbracket$-modules are understood to be completed in the $\hbar$-adic topology. For $p \in \mathbb{N}$, let $a_{i j}=2 \delta_{i j}-\delta_{|i-j|=1}, 1 \leq i, j \leq p$, be the entries of the Cartan matrix 
of type $A_{p-1}$ and let $U_{\hbar} \mathfrak{g l}_{p}$ be the corresponding Drinfeld-Jimbo quantum group [Dr1, Ji1] i.e., the algebra over $\mathbb{C} \llbracket \hbar \rrbracket$ topologically generated by elements $E_{i}, F_{i}, i=1 \ldots p-1$ and $D_{i}$, $i=1 \ldots p$ subject to the $q$-Serre relations

$$
\begin{gathered}
{\left[D_{i}, D_{j}\right]=0} \\
{\left[D_{i}, E_{j}\right]=\left(\delta_{i j}-\delta_{i j+1}\right) E_{j} \quad\left[D_{i}, F_{j}\right]=-\left(\delta_{i j}-\delta_{i j+1}\right) F_{j}} \\
{\left[E_{i}, F_{j}\right]=\delta_{i j} \frac{e^{\hbar H_{i}}-e^{-\hbar H_{i}}}{e^{\hbar}-e^{-\hbar}}} \\
\sum_{k=0}^{1-a_{i j}}(-1)^{k}\left[\begin{array}{c}
1-a_{i j} \\
k
\end{array}\right] E_{i}^{k} E_{j} E_{i}^{1-a_{i j}-k}=0, \quad \forall i \neq j \\
\sum_{k=0}^{1-a_{i j}}(-1)^{k}\left[\begin{array}{c}
1-a_{i j} \\
k
\end{array}\right] F_{i}^{k} F_{j} F_{i}^{1-a_{i j}-k}=0, \quad \forall i \neq j
\end{gathered}
$$

where $H_{i}=D_{i}-D_{i+1}$ and, for any $n \geq k \in \mathbb{N}$,

$$
\begin{aligned}
{[n] } & =\frac{e^{n \hbar}-e^{-n \hbar}}{e^{\hbar}-e^{\hbar}} \\
{[n] ! } & =[n][n-1] \cdots[1] \\
{\left[\begin{array}{l}
n \\
k
\end{array}\right] } & =\frac{[n] !}{[k] ![n-k] !}
\end{aligned}
$$

$U_{\hbar} \mathfrak{g l}_{p}$ is a topological Hopf algebra with coproduct $\Delta$ and counit $\varepsilon$ given by

$$
\begin{gathered}
\Delta\left(D_{i}\right)=D_{i} \otimes 1+1 \otimes D_{i} \\
\Delta\left(E_{i}\right)=E_{i} \otimes e^{\hbar H_{i}}+1 \otimes E_{i} \\
\Delta\left(F_{i}\right)=F_{i} \otimes 1+e^{-\hbar H_{i}} \otimes F_{i}
\end{gathered}
$$

and

$$
\varepsilon\left(E_{i}\right)=\varepsilon\left(F_{i}\right)=\varepsilon\left(D_{i}\right)=0
$$

Note that $I=D_{1}+\cdots+D_{p}$ is central so that $U_{\hbar} \mathfrak{g l}_{p} \cong U_{\hbar \mathfrak{s l}} \otimes \mathbb{C}[I] \llbracket \hbar \rrbracket$ as Hopf algebras where the coproduct on $\mathbb{C}[I] \llbracket \hbar \rrbracket$ is given by $\Delta(I)=I \otimes 1+1 \otimes I$ and $U_{\hbar \mathfrak{s l}} \subset U_{\hbar \mathfrak{g l}_{p}}$ is the closed Hopf subalgebra generated by $E_{i}, F_{i}$ and $H_{i}, i=1 \ldots p-1$.

By a finite-dimensional representation of $U_{\hbar} \mathfrak{g l}_{p}$ we shall mean a $U_{\hbar} \mathfrak{g l}_{p}$-module which is topologically free and finitely generated over $\mathbb{C} \llbracket \hbar \rrbracket$ and on which $I$ acts semisimply with eigenvalues in $\mathbb{C}$. Choose an algebra isomorphism $\phi: U_{\hbar} \mathfrak{g l}_{p} \rightarrow U \mathfrak{g l}_{p} \llbracket \hbar \rrbracket$ mapping each $D_{i}$ onto $E_{i i}$ Dr2, prop. 4.3] and let $V$ be a finite-dimensional $\mathfrak{g l}_{p}$-module on which $1^{(p)}=\sum_{i=1}^{p} E_{i i}$ acts semisimply. Then, $U \mathfrak{g l}_{p} \llbracket \hbar \rrbracket$ acts on $V \llbracket \hbar \rrbracket$ and the latter becomes, via $\phi$, a finite-dimensional representation of $U_{\hbar} \mathfrak{g l}_{p}$. Conversely,

Proposition 4.1. Let $\mathcal{V}$ be a finite-dimensional representation of $U_{\hbar} \mathfrak{g l}_{p}$ and $V=\mathcal{V} / \hbar V$ the corresponding $\mathfrak{g l}_{p}-$ module. Then, as $U_{\hbar} \mathfrak{g l}_{p}$-modules,

$$
\mathcal{V} \cong V \llbracket \hbar \rrbracket
$$

Proof. Since $I$ is diagonalisable on $\mathcal{V}$ and commutes with $U_{\hbar} \mathfrak{g l}_{p}$, we may assume that it acts on $\mathcal{V}$ as multiplication by a scalar $\lambda \in \mathbb{C}$. Since $\mathcal{V}$ is topologically free, $\mathcal{V} \cong V \llbracket \hbar \rrbracket$ as $\mathbb{C} \llbracket \hbar \rrbracket$-modules so that $\mathcal{V}$ is a deformation of the finite-dimensional $\mathfrak{s l}_{p}$-module $V$. Since $\mathfrak{s l}_{p}$ is 
simple, $H^{1}\left(\mathfrak{s l}_{p}, V\right)=0$ and $\mathcal{V}$ is isomorphic, as $\mathfrak{s l}_{p}$, and therefore as $\mathfrak{g l}_{p}$-module to the trivial deformation of $V$. Thus, $\mathcal{V} \cong V \llbracket \hbar \rrbracket$ as $U \mathfrak{g l}_{p} \llbracket \hbar \rrbracket$, and therefore as $U_{\hbar} \mathfrak{g l}_{p}-$ modules

Corollary 4.2. Let $U, V$ be finite-dimensional $\mathfrak{g l}_{p}$-modules on which $1^{(p)}$ acts semisimply. If $U \otimes V$ decomposes as

$$
U \otimes V \cong \bigoplus_{W} N_{W} W
$$

for some $\mathfrak{g l}_{p}-$ modules $W$ and multiplicities $N_{W} \in \mathbb{N}$, then, as $U_{\hbar} \mathfrak{g l}_{p}-$ modules,

$$
U \llbracket \hbar \rrbracket \otimes \llbracket \hbar \rrbracket \cong \bigoplus_{W} N_{W} W \llbracket \hbar \rrbracket
$$

Proof. By (4.14), both sides of (4.15) have the same specialisation at $\hbar=0$ and are therefore isomorphic by proposition 4.1

\section{The DUAL PAIR $\left(U_{\hbar} \mathfrak{g l}_{k}, U_{\hbar} \mathfrak{g l}_{n}\right)$}

We shall need the analogue of theorem 3.2 in the setting of the algebra $\mathcal{S}_{\hbar}\left(\mathcal{M}_{k, n}^{*}\right)$ of functions on quantum $k \times n$ matrix space. With the exception of theorems 5.4 and 5.5, this section follows [Ba, §1.5] (see also Ga $)$. By definition, $\mathcal{S}_{\hbar}\left(\mathcal{M}_{k, n}^{*}\right)$ is the algebra over $\mathbb{C} \llbracket \hbar \rrbracket$ topologically generated by elements $X_{i j}, 1 \leq i \leq k, 1 \leq j \leq n$ with relations

$$
X_{i j} X_{k l}=\left\{\begin{array}{cl}
X_{k l} X_{i j} & \text { if } k>i \text { and } l<j \text { or } k<i \text { and } l>j \\
e^{-\hbar} X_{k l} X_{i j} & \text { if } k>i \text { and } l=j \text { or } k=i \text { and } l>j \\
X_{k l} X_{i j}-\left(e^{\hbar}-e^{-\hbar}\right) X_{k j} X_{i l} & \text { if } k>i \text { and } l>j
\end{array}\right.
$$

$\mathcal{S}_{\hbar}\left(\mathcal{M}_{k, n}^{*}\right)$ is $\mathbb{N}$-graded by decreeing that each $X_{i j}$ is of degree 1 and we denote its homogeneous components by $\mathcal{S}_{\hbar}^{d}\left(\mathcal{M}_{k, n}^{*}\right), d \in \mathbb{N}$. For any $k \times n$ matrix $m$ with entries $m_{i j} \in \mathbb{N}$, set

$$
\begin{aligned}
\mathbf{X}^{m} & =X_{11}^{m_{11}} \cdots X_{k 1}^{m_{k 1}} \cdots X_{1 n}^{m_{1 n}} \cdots X_{k n}^{m_{k n}} \\
& =X_{11}^{m_{11}} \cdots X_{1 n}^{m_{1 n}} \cdots X_{k 1}^{m_{k 1}} \cdots X_{k n}^{m_{k n}}
\end{aligned}
$$

By the commutation relations (5.1), the $\mathbf{X}^{m}$, with $m \in \mathcal{M}_{k, n}$ such that $|m|=d$, span $\mathcal{S}_{\hbar}^{d}\left(\mathcal{M}_{k, n}^{*}\right)$, where $|m|=\sum_{i, j} m_{i j}$.

Theorem 5.1 (Parshall-Wang). The monomials $\mathbf{X}^{m}, m \in \mathcal{M}_{k n}(\mathbb{N})$, are linearly independent over $\mathbb{C} \llbracket \hbar \rrbracket$. In particular, the set $\left\{\mathbf{X}^{m}\right\}_{|m|=d}$ is a $\mathbb{C} \llbracket \hbar \rrbracket$-basis of $\mathcal{S}_{\hbar}^{d}\left(\mathcal{M}_{k, n}^{*}\right)$.

Proof. This is proved in $[\mathrm{PW}$, thm. 3.5.1] for $k=n$ and over the field $\mathbb{C}(q)$ of rational functions of $q=e^{\hbar}$ rather than over $\mathbb{C} \llbracket \hbar \rrbracket$. The proof however works equally well for $k \neq n$ and, as remarked in $[\mathrm{PW}]$, over $\mathbb{C} \llbracket \hbar \rrbracket$

As in the classical case, $\mathcal{S}_{\hbar}\left(\mathcal{M}_{k, n}^{*}\right)$ is a module algebra over $U_{\hbar} \mathfrak{g l}_{k} \otimes U_{\hbar} \mathfrak{g l}_{n}$. This may be seen in the following way. For any $l \in \mathbb{N}$, one readily checks that the assignement

$$
X_{i j} \rightarrow \sum_{l^{\prime}=1}^{l} X_{i l^{\prime}} \otimes X_{l^{\prime} j}
$$


extends uniquely to an algebra homomorphism $\Delta_{k l n}: \mathcal{S}_{\hbar}\left(\mathcal{M}_{k, n}^{*}\right) \rightarrow \mathcal{S}_{\hbar}\left(\mathcal{M}_{k, l}^{*}\right) \otimes \mathcal{S}_{\hbar}\left(\mathcal{M}_{l, n}^{*}\right)$ such that, for any $l, m \in \mathbb{N}$, the following diagram commutes

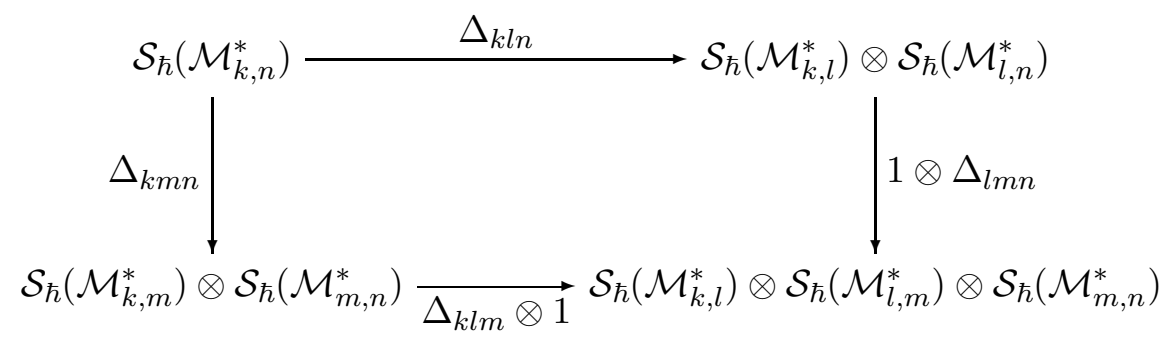

In particular, $\mathcal{S}_{\hbar}\left(\mathcal{M}_{k, k}^{*}\right)$ and $\mathcal{S}_{\hbar}\left(\mathcal{M}_{n, n}^{*}\right)$ are topological bialgebras with comultiplications $\Delta_{k k k}$ and $\Delta_{n n n}$ respectively and counit $\varepsilon\left(X_{i j}\right)=\delta_{i j}$. Moreover, the maps $\Delta_{k k n}$ and $\Delta_{k n n}$ give $\mathcal{S}_{\hbar}\left(\mathcal{M}_{k, n}^{*}\right)$ the structure of a $\mathcal{S}_{\hbar}\left(\mathcal{M}_{k, k}^{*}\right)-\mathcal{S}_{\hbar}\left(\mathcal{M}_{n, n}^{*}\right)$ bicomodule algebra each homogeneous component of which is invariant under $\mathcal{S}_{\hbar}\left(\mathcal{M}_{k, k}^{*}\right)$ and $\mathcal{S}_{\hbar}\left(\mathcal{M}_{n, n}^{*}\right)$ since $\Delta_{k l n}\left(\mathcal{S}_{\hbar}^{d}\left(\mathcal{M}_{k, k}\right)\right) \subset$ $\mathcal{S}_{\hbar}^{d}\left(\mathcal{M}_{k, k}\right) \otimes \mathcal{S}_{\hbar}^{d}\left(\mathcal{M}_{l, l}\right)$.

We shall need a columnwise (resp. rowwise) description of the coaction of $\mathcal{S}_{\hbar}\left(\mathcal{M}_{k, k}^{*}\right)$ (resp. $\left.\mathcal{S}_{\hbar}\left(\mathcal{M}_{n, n}^{*}\right)\right)$ on $\mathcal{S}_{\hbar}\left(\mathcal{M}_{k, n}^{*}\right)$. Consider the quantum $k$ and $n$-dimensional planes i.e., the algebras $\mathcal{S}_{\hbar}\left(\mathcal{M}_{k, 1}^{*}\right)$ and $\mathcal{S}_{\hbar}\left(\mathcal{M}_{1, n}^{*}\right)$. By the commutation relations (5.1) and theorem 5.1, these may be embedded as subalgebras of $\mathcal{S}_{\hbar}\left(\mathcal{M}_{k, n}^{*}\right)$ via the maps

$$
\begin{array}{ll}
c_{j}: \mathcal{S}_{\hbar}\left(\mathcal{M}_{k, 1}^{*}\right) \rightarrow \mathcal{S}_{\hbar}\left(\mathcal{M}_{k, n}^{*}\right), & c_{j}\left(X_{i 1}\right)=X_{i j} \\
r_{i}: \mathcal{S}_{\hbar}\left(\mathcal{M}_{1, n}^{*}\right) \rightarrow \mathcal{S}_{\hbar}\left(\mathcal{M}_{k, n}^{*}\right), & r_{i}\left(X_{1 j}\right)=X_{i j}
\end{array}
$$

with $1 \leq i \leq k, 1 \leq j \leq n$. By $($ 5.4 $), \mathcal{S}_{\hbar}\left(\mathcal{M}_{k, 1}^{*}\right)$ is a left algebra comodule over $\mathcal{S}_{\hbar}\left(\mathcal{M}_{k, k}^{*}\right)$ and $\mathcal{S}_{\hbar}\left(\mathcal{M}_{1, n}^{*}\right)$ a right algebra comodule over $\mathcal{S}_{\hbar}\left(\mathcal{M}_{n, n}^{*}\right)$.

Lemma 5.2. As left, $\mathbb{N}$-graded $\mathcal{S}_{\hbar}\left(\mathcal{M}_{k, k}^{*}\right)$-comodules,

$$
\mathcal{S}_{\hbar}\left(\mathcal{M}_{k, n}^{*}\right) \cong \mathcal{S}_{\hbar}\left(\mathcal{M}_{k, 1}^{*}\right)^{\otimes n}
$$

via the map $\Phi: p_{1} \otimes \cdots \otimes p_{n} \rightarrow c_{1}\left(p_{1}\right) \cdots c_{n}\left(p_{n}\right)$. Similarly, as right, $\mathbb{N}$-graded $\mathcal{S}_{\hbar}\left(\mathcal{M}_{n, n}^{*}\right)$ comodules,

$$
\mathcal{S}_{\hbar}\left(\mathcal{M}_{k, n}^{*}\right) \cong \mathcal{S}_{\hbar}\left(\mathcal{M}_{1, n}^{*}\right)^{\otimes k}
$$

via $\Psi: q_{1} \otimes \cdots \otimes q_{k} \rightarrow r_{1}\left(q_{1}\right) \cdots r_{k}\left(q_{k}\right)$.

Proof. The map $\Phi$ clearly preserves the grading and, by theorem 5.1, restricts to a $\mathbb{C} \llbracket \hbar \rrbracket-$ linear isomorphism of homogeneous components since it bijectively maps the monomial basis of $\mathcal{S}_{\hbar}\left(\mathcal{M}_{k, 1}^{*}\right)^{\otimes n}$ onto the basis $\mathbf{X}^{m}$ of $\mathcal{S}_{\hbar}\left(\mathcal{M}_{k, n}^{*}\right)$. The fact that $\Phi$ itself is an isomorphism follows easily because any element of $\mathcal{S}_{\hbar}\left(\mathcal{M}_{1, n}^{*}\right)^{\otimes k}$ or $\mathcal{S}_{\hbar}\left(\mathcal{M}_{k, n}^{*}\right)$ is the convergent sum of its homogeneous components. As readily checked, the diagram

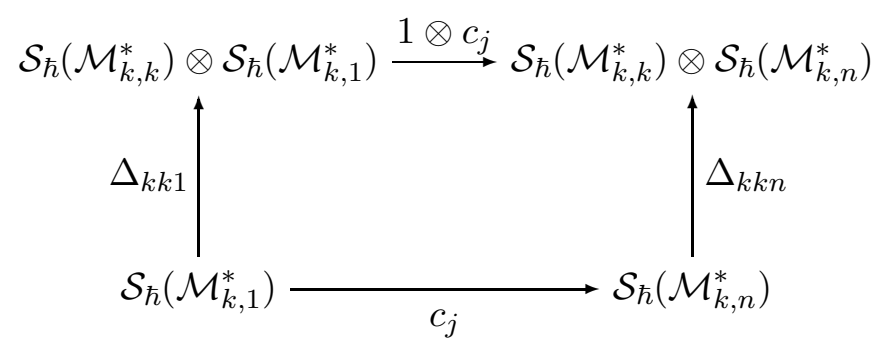


is commutative for any $1 \leq j \leq n$ and therefore so is

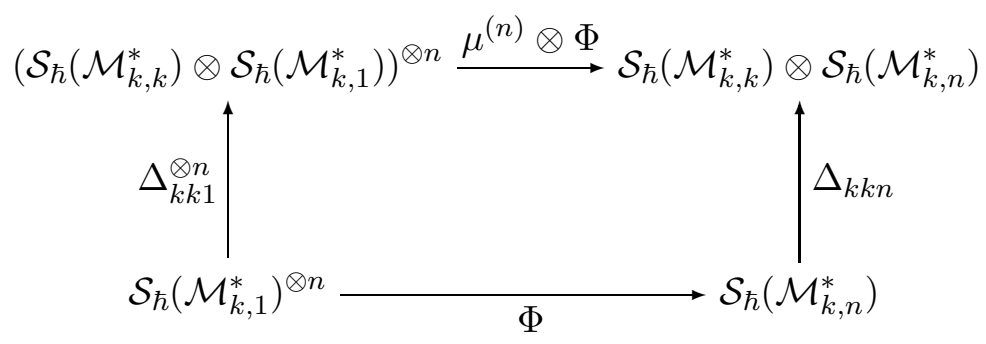

where $\mu^{(n)}: \mathcal{S}_{\hbar}\left(\mathcal{M}_{k, k}^{*}\right)^{\otimes n} \rightarrow \mathcal{S}_{\hbar}\left(\mathcal{M}_{k, k}^{*}\right)$ is the $n$-fold multiplication. This proves (5.7). The proof of (5.8) is identical

We turn now to the action of $U_{\hbar} \mathfrak{g l}_{k} \otimes U_{\hbar} \mathfrak{g l}_{n}$ on $\mathcal{S}_{\hbar}\left(\mathcal{M}_{k, n}^{*}\right)$. For $p=k, n$, consider the vector representation of $U_{\hbar} \mathfrak{g l}_{p}$ i.e., the module $V=\mathbb{C}^{p} \llbracket \hbar \rrbracket$ with basis $e_{1}, \ldots e_{p}$ and action given by

$$
D_{i}=E_{i i}, \quad E_{i}=E_{i i+1}, \quad F_{i}=E_{i+1 i}
$$

where $E_{a b} e_{c}=\delta_{b c} e_{a}$. Let $e^{1}, \ldots, e^{p} \in V^{*}$ be the dual basis of $e_{1}, \ldots, e_{p}, U_{\hbar \mathfrak{g}} \mathfrak{l}_{p}^{\circ}$ the restricted dual of $U_{\hbar} \mathfrak{g l}_{p}$ and $t_{i j} \in U_{\hbar} \mathfrak{g l}_{p}^{\circ}$ the matrix coefficient defined by

$$
t_{i j}(x)=\left\langle e^{i}, x e_{j}\right\rangle
$$

Proposition 5.3. The assignement $X_{i j} \rightarrow t_{i j}$ extends uniquely to a bialgebra morphism $\kappa_{p}: \mathcal{S}_{\hbar}\left(\mathcal{M}_{p, p}^{*}\right) \rightarrow U_{\hbar} \mathfrak{g l}_{p}^{\circ}$.

Proof. We need to check that the $t_{i j}$ satisfy the relations (5.1), i.e., that when evaluated on $\Delta(x), x \in U_{\hbar} \mathfrak{g l}_{p}$,

$$
t_{i j} \otimes t_{k l}=\left\{\begin{array}{cl}
t_{k l} \otimes t_{i j} & \text { if } k>i \text { and } l<j \text { or } k<i \text { and } l>j \\
e^{-\hbar} t_{k l} \otimes t_{i j} & \text { if } k>i \text { and } l=j \text { or } k=i \text { and } l>j \\
t_{k l} \otimes t_{i j}-\left(e^{\hbar}-e^{-\hbar}\right) t_{k j} \otimes t_{i l} & \text { if } k>i \text { and } l>j
\end{array}\right.
$$

Let $R^{\vee}=\sigma \cdot R \in \operatorname{End}(V \otimes V)$ where $\sigma \in G L(V \otimes V)$ is the flip and $R$ is the universal $R$-matrix of $U_{\hbar \mathfrak{g l}_{p}}$ acting on $V \otimes V$. Then [Ji2], [CP, §8.3.G]

$$
R=\left(e^{\hbar} \sum_{i=1}^{p} E_{i i} \otimes E_{i i}+\sum_{1 \leq i \neq j \leq p} E_{i i} \otimes E_{j j}+\left(e^{\hbar}-e^{-\hbar}\right) \sum_{1 \leq i<j \leq p} E_{i j} \otimes E_{j i}\right)
$$

so that the matrix entries of $R^{\vee}$ are

$$
R_{i k, j l}^{\vee}=\left\{\begin{array}{cl}
e^{\delta_{j l} \hbar} & \text { if } i=l \text { and } k=j \\
e^{\hbar}-e^{-\hbar} & \text { if } i=j, k=l \text { and } j>l \\
0 & \text { otherwise }
\end{array}\right.
$$

From (5.15), one readily checks that both sides of 5.13 coincide when evaluated on any $A \in \operatorname{End}(V \otimes V)$ commuting with $R^{\vee}$. Since $R^{\vee}$ is a $U_{\hbar} \mathfrak{g l}_{p}$-intertwiner, $\kappa$ extends to an algebra morphism which respects the counit and coproduct since $\Delta\left(t_{i j}\right)=\sum_{q=1}^{p} t_{i q} \otimes t_{q j}$

\section{Theorem 5.4.}

1. The maps $\kappa_{p}, p=k, n$ of proposition 5.3 give $\mathcal{S}_{\hbar}\left(\mathcal{M}_{k, n}^{*}\right)$ the structure of an algebra module over $U_{\hbar} \mathfrak{g l}_{k} \otimes U_{\hbar} \mathfrak{g l}_{n}$ with invariant homogeneous components $\mathcal{S}_{\hbar}^{d}\left(\mathcal{M}_{k, n}^{*}\right), d \in \mathbb{N}$. 
2. The maps $\Phi, \Psi$ of lemma 5.2 yield isomorphisms

$$
\mathcal{S}_{\hbar}\left(\mathcal{M}_{k, n}^{*}\right) \cong \mathcal{S}_{\hbar}\left(\mathcal{M}_{k, 1}^{*}\right)^{\otimes n} \quad \text { and } \quad \mathcal{S}_{\hbar}\left(\mathcal{M}_{k, n}^{*}\right) \cong \mathcal{S}_{\hbar}\left(\mathcal{M}_{1, n}^{*}\right)^{\otimes k}
$$

as $\mathbb{N}$-graded $U_{\hbar} \mathfrak{g l}_{k}$ and $U_{\hbar} \mathfrak{g l}_{n}$-modules respectively.

3. The action of the generators $E_{q}^{(p)}, F_{q}^{(p)}, q=1 \ldots p-1$ and $D_{q}^{(p)}, q=1 \ldots p$ of $U_{\hbar} \mathfrak{g l}_{p}$, $p=k, n$ in the monomial basis $\mathbf{X}^{m}, m \in \mathcal{M}_{k, n}$, is given by

$$
\begin{aligned}
& D_{i}^{(k)} \mathbf{X}^{m}=\sum_{j=1}^{n} m_{i j} \mathbf{X}^{m} \\
& E_{i}^{(k)} \mathbf{X}^{m}=\sum_{j=1}^{n}\left[m_{i+1 j}\right] \prod_{j^{\prime}=j+1}^{n} e^{\hbar\left(m_{i j^{\prime}}-m_{i+1 j^{\prime}}\right)} \mathbf{X}^{m+\varepsilon_{i j}-\varepsilon_{i+1 j}} \\
& F_{i}^{(k)} \mathbf{X}^{m}=\sum_{j=1}^{n}\left[m_{i j}\right] \prod_{j^{\prime}=1}^{j-1} e^{-\hbar\left(m_{i j^{\prime}}-m_{i+1 j^{\prime}}\right)} \mathbf{X}^{m-\varepsilon_{i j}+\varepsilon_{i+1 j}}
\end{aligned}
$$

where $\left(\varepsilon_{a b}\right)_{c d}=\delta_{a c} \delta_{b d}$, and

$$
\begin{aligned}
& D_{j}^{(n)} \mathbf{X}^{m}=\sum_{i=1}^{k} m_{i j} \mathbf{X}^{m} \\
& E_{j}^{(n)} \mathbf{X}^{m}=\sum_{i=1}^{k}\left[m_{i j+1}\right] \prod_{i^{\prime}=i+1}^{k} e^{\hbar\left(m_{i^{\prime} j}-m_{i^{\prime} j+1}\right)} \mathbf{X}^{m+\varepsilon_{i j}-\varepsilon_{i j+1}} \\
& F_{j}^{(n)} \mathbf{X}^{m}=\sum_{i=1}^{k}\left[m_{i j}\right] \prod_{i^{\prime}=1}^{i-1} e^{-\hbar\left(m_{i^{\prime} j}-m_{i^{\prime} j+1}\right)} \mathbf{X}^{m-\varepsilon_{i j}+\varepsilon_{i j+1}}
\end{aligned}
$$

Proof. Using the transposition anti-involution $\tau$ on $\mathcal{S}_{\hbar}\left(\mathcal{M}_{k, k}^{*}\right)$ given by $\tau\left(X_{i j}\right)=X_{j i}$, we may regard $\mathcal{S}_{\hbar}\left(\mathcal{M}_{k, n}^{*}\right)$ as a right algebra comodule over $\mathcal{S}_{\hbar}\left(\mathcal{M}_{k, k}^{*}\right) \otimes \mathcal{S}_{\hbar}\left(\mathcal{M}_{n, n}^{*}\right)$ and therefore, via the pairings $\langle\cdot, \cdot\rangle: \mathcal{S}_{\hbar}\left(\mathcal{M}_{m, m}^{*}\right) \otimes U_{\hbar} \mathfrak{g l}_{m} \rightarrow \mathbb{C} \llbracket \hbar \rrbracket, m=k, n$, given by proposition 5.3 as a left algebra module over $U_{\hbar} \mathfrak{g l}_{k} \otimes U_{\hbar} \mathfrak{g l}_{n}$. This proves (i) and (ii). Explicitly, for $x^{(m)} \in U_{\hbar} \mathfrak{g l}_{m}$, $m=k, n$ and $p \in \mathcal{S}_{\hbar}\left(\mathcal{M}_{k, n}^{*}\right)$

$$
\begin{aligned}
x^{(k)} p & =\left\langle x^{(k)} \otimes 1, \tau \otimes 1 \cdot \Delta_{k k n}(p)\right\rangle \\
x^{(n)} p & =\left\langle 1 \otimes x^{(n)}, \Delta_{k n n}(p)\right\rangle
\end{aligned}
$$

Using (5.23) and (5.11), one gets

$$
\begin{aligned}
& D_{i}^{(k)} X_{i^{\prime} j}=\delta_{i i^{\prime}} X_{i j} \\
& E_{i}^{(k)} X_{i^{\prime} j}=\delta_{i+1 i^{\prime}} X_{i j} \\
& F_{i}^{(k)} X_{i^{\prime} j}=\delta_{i i^{\prime}} X_{i+1 j}
\end{aligned}
$$

Using the algebra module property $x(p q)=\mu(\Delta(x) p \otimes q)$ where $x \in U_{\hbar} \mathfrak{g l}_{k}, p, q \in \mathcal{S}_{\hbar}\left(\mathcal{M}_{k, n}^{*}\right)$ and $\mu: \mathcal{S}_{\hbar}\left(\mathcal{M}_{k, n}^{*}\right)^{\otimes 2} \rightarrow \mathcal{S}_{\hbar}\left(\mathcal{M}_{k, n}^{*}\right)$ is multiplication, (4.9)-(4.11) and the commutation relations (5.1) shows by induction on $m \in \mathbb{N}$ that

$$
\begin{aligned}
D_{i}^{(k)} X_{i^{\prime} j}^{m} & =\delta_{i i^{\prime}} m X_{i j}^{m} \\
E_{i}^{(k)} X_{i^{\prime} j}^{m} & =\delta_{i+1 i^{\prime}}[m] X_{i j} X_{i+1 j}^{m-1} \\
F_{i}^{(k)} X_{i^{\prime} j}^{m} & =\delta_{i i^{\prime}}[m] X_{i j}^{m-1} X_{i+1 j}
\end{aligned}
$$


Let $\Delta^{(a)}: U_{\hbar} \mathfrak{g l}_{k} \rightarrow U_{\hbar} \mathfrak{g l}_{k}^{\otimes a}, a \in \mathbb{N}^{*}$ be recursively defined by $\Delta^{(1)}=\mathrm{id}, \Delta^{(a+1)}=\Delta \otimes$ $\mathrm{id}^{\otimes(a-1)} \cdot \Delta^{(a)}$. Then, by $(4.9)-(4.11)$

$$
\begin{aligned}
& \Delta^{(a)} D_{i}^{(k)}=\sum_{b=1}^{a} 1^{\otimes(b-1)} \otimes D_{i}^{(k)} \otimes 1^{\otimes(a-b)} \\
& \Delta^{(a)} E_{i}^{(k)}=\sum_{b=1}^{a} 1^{\otimes(b-1)} \otimes E_{i}^{(k)} \otimes\left(e^{\hbar H_{i}^{(k)}}\right)^{\otimes(a-b)} \\
& \Delta^{(a)} F_{i}^{(k)}=\sum_{b=1}^{a}\left(e^{-\hbar H_{i}^{(k)}}\right)^{\otimes(b-1)} \otimes F_{i}^{(k)} \otimes 1^{\otimes(a-b)}
\end{aligned}
$$

The formulae $(5.17)-(\sqrt[5.19]{)})$ now follow from the algebra module property and (5.31) $-(5.33)$. The proof of (5.20)-(5.22) is similar

The following result is proved in [Ba] and [Ga] for the quantum groups $U_{q} \mathfrak{g l}_{k}, U_{q} \mathfrak{g l}_{n}$ by a different method

Theorem 5.5. For any $d \in \mathbb{N}$, the $U_{\hbar} \mathfrak{g l}_{k} \otimes U_{\hbar} \mathfrak{g l}_{n}$-module $\mathcal{S}_{\hbar}^{d}\left(\mathcal{M}_{k, n}^{*}\right)$ decomposes as

$$
\mathcal{S}_{\hbar}^{d}\left(\mathcal{M}_{k, n}^{*}\right) \cong \bigoplus_{\substack{\lambda \in \mathbb{Y}_{\min (k, n)} \\|\lambda|=d}} V_{\lambda}^{(k)} \llbracket \hbar \rrbracket \otimes V_{\lambda}^{(n)} \llbracket \hbar \rrbracket
$$

Proof. By theorem 5.1, $\mathcal{S}_{\hbar}^{d}\left(\mathcal{M}_{k, n}^{*}\right)$ has no torsion, and is therefore a topologically free $\mathbb{C} \llbracket \hbar \rrbracket-$ module. Moreover, by (5.17)-(5.22), $\mathcal{S}_{\hbar}^{d}\left(\mathcal{M}_{k, n}^{*}\right) / \hbar \mathcal{S}_{\hbar}^{d}\left(\mathcal{M}_{k, n}^{*}\right)$ is the $\mathfrak{g l}_{k} \oplus \mathfrak{g l}_{n}-$ module $\mathcal{S}^{d}\left(\mathcal{M}_{k, n}^{*}\right)$. The conclusion follows from theorem 3.2 and proposition 4.1

\section{Braid Group aCtions on QUANTUM Matrix SPACE}

We compare in this section two actions of the braid group $B_{n}$ on the algebra $\mathcal{S}_{\hbar}\left(\mathcal{M}_{k, n}^{*}\right)$ of functions of quantum $k \times n$ matrix space. The first is the $R$-matrix representation obtained by regarding $\mathcal{S}_{\hbar}\left(\mathcal{M}_{k, n}^{*}\right)$ as the $U_{\hbar} \mathfrak{g l}_{k}-$ module $\mathcal{S}_{\hbar}\left(\mathcal{M}_{k, 1}^{*}\right)^{\otimes n}$. The second is the quantum Weyl group action of $B_{n}$ on $\mathcal{S}_{\hbar}\left(\mathcal{M}_{k, n}^{*}\right)$ viewed as a $U_{\hbar} \mathfrak{g l}_{n}$-module. We will show that these representations essentially coincide, thus extending to the $q$-setting the fact that the symmetric group $\mathfrak{S}_{n}$ acts on $\left(S^{\bullet} \mathbb{C}^{k}\right)^{\otimes n} \cong \mathcal{S}\left(\mathcal{M}_{k, n}^{*}\right)$ via the permutation matrices in $G L_{n}(\mathbb{C})$.

More precisely, for any $1 \leq j \leq n$, let $R_{j}^{\vee}$ be the universal $R$-matrix of $U_{\hbar} \mathfrak{g l}_{k}$ acting on the $j$ and $j+1$ tensor copies of $\mathcal{S}_{\hbar}\left(\mathcal{M}_{k, n}^{*}\right) \cong \mathcal{S}_{\hbar}\left(\mathcal{M}_{k, 1}^{*}\right)^{\otimes n}$ and $S_{j}$ the quantum Weyl group element of $U_{\hbar} \mathfrak{g l}_{n}$ corresponding to the simple root $\alpha_{j}=\theta_{j}-\theta_{j+1}$. We will show that

$$
R_{j}^{\vee}=S_{j} \cdot e^{-\hbar\left(D_{j}^{(n)}+D_{j}^{(n)} D_{j+1}^{(n)} / k\right)} \cdot e^{i \pi D_{j}^{(n)}}
$$

where $D_{1}^{(n)}, \ldots, D_{n}^{(n)}$ are the generators of the Cartan subalgebra of $U_{\hbar} \mathfrak{g l}_{n}$. The proof of (6.1) is based upon the following observation, which we owe to B. Feigin. Both sides of 6.1 only act upon the $j$ and $j+1$ tensor copies of $\mathcal{S}_{\hbar}\left(\mathcal{M}_{k, 1}^{*}\right)^{\otimes n}$ so that its proof reduces to a computation in $\mathcal{S}_{\hbar}\left(\mathcal{M}_{k, 1}^{*}\right)^{\otimes 2} \cong \mathcal{S}_{\hbar}\left(\mathcal{M}_{k, 2}^{*}\right)$. Since both sides intertwine the action of $U_{\hbar} \mathfrak{g l}_{k}$ on $\mathcal{S}_{\hbar}\left(\mathcal{M}_{k, 2}^{*}\right)$, it suffices to compare them on highest weight vectors. These, and the action of $R_{j}^{\vee}$ are computed in $\S 6.1$. The action of $S_{j}$ is computed in $\$ 6.2$. 
REMARK. It is easy to check that neither action of $B_{n}$ is compatible with the algebra structure of $\mathcal{S}_{\hbar}\left(\mathcal{M}_{k, n}^{*}\right)$, so that (6.1) cannot be proved by merely checking it on the generators $X_{i j}$. This stems from the fact that quantum Weyl group operators are not group-like.

6.1. $R$-matrix action on singular vectors. For any $d \in \mathbb{N}$, let $\mathcal{S}_{\hbar}^{d} \mathbb{C}^{k}$ be the homogeneous component of degree $d$ of $\mathcal{S}_{\hbar}\left(\mathcal{M}_{k, 1}^{*}\right)$. By (5.17)-(5.19), $\mathcal{S}_{\hbar}^{d} \mathbb{C}^{k}$ is a deformation of the $d$-th symmetric power $\mathcal{S}^{d} \mathbb{C}^{k}$ of the vector representation of $\mathfrak{g l}_{k}$. Let $\mu_{1}, \mu_{2} \in \mathbb{N}$, then

Lemma 6.1. As $U_{\hbar} \mathfrak{g l}_{k}-$ modules,

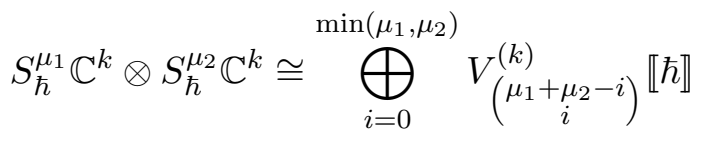

where $V_{\left(\begin{array}{c}(a) \\ b\end{array}\right)}^{(k)}$ is the irreducible representation of $\mathfrak{g l}_{k}$ with highest weight $(a, b, 0, \ldots, 0)$. The corresponding highest weight vectors $v_{i}^{\mu_{1}, \mu_{2}}$ are given by

$$
v_{i}^{\mu_{1}, \mu_{2}}=\sum_{a=0}^{i}(-1)^{a}\left[\begin{array}{l}
i \\
a
\end{array}\right] e^{\hbar a\left(\mu_{2}-a+1\right)} X_{11}^{\mu_{1}-i+a} X_{21}^{i-a} \otimes X_{12}^{\mu_{2}-a} X_{22}^{a}
$$

Proof. The decomposition (6.2) follows from the Pieri rules for $\mathfrak{g l}_{k}$ and corollary 4.2. Fix $i \in\left\{0, \ldots, \min \left(\mu_{1}, \mu_{2}\right)\right\}$. By (5.17), any $v \in S_{\hbar}^{\mu_{1}} \mathbb{C}^{k} \otimes S_{\hbar}^{\mu_{2}} \mathbb{C}^{k}$ of weight $\left(\mu_{1}+\mu_{2}-i, i, 0 \ldots, 0\right)$ is of the form

$$
v=\sum_{a=0}^{i} c_{a} X_{11}^{\mu_{1}-i+a} X_{21}^{i-a} \otimes X_{12}^{\mu_{2}-a} X_{22}^{a}
$$

for some constants $c_{a} \in \mathbb{C}$. By $(4.10)$ and (5.18), $\Delta\left(E_{j}\right) v=0$ for any $j \geq 2$ so that $v$ is a highest weight vector iff

$$
\begin{aligned}
\Delta\left(E_{1}\right) v & =\sum_{a=0}^{i} c_{a}[i-a] e^{\hbar\left(\mu_{2}-2 a\right)} X_{11}^{\mu_{1}-i+a+1} X_{21}^{i-a-1} \otimes X_{12}^{\mu_{2}-a} X_{22}^{a} \\
& +\sum_{a=0}^{i} c_{a}[a] X_{11}^{\mu_{1}-i+a} X_{21}^{i-a} \otimes X_{12}^{\mu_{2}-a+1} X_{22}^{a-1}
\end{aligned}
$$

is equal to zero. This yields $c_{a}=-c_{a-1} e^{\hbar\left(\mu_{2}-2 a+2\right)}[i-a+1] /[a]$ and therefore

$$
c_{a}=(-1)^{a}\left[\begin{array}{l}
i \\
a
\end{array}\right] e^{\hbar a\left(\mu_{2}-a+1\right)} c_{0}
$$

whence 6.3$)$

Let $R$ be the universal $R$-matrix of $U_{\hbar} \mathfrak{s l}_{k}$ and $R^{\vee}=\sigma \cdot R: S_{\hbar}^{\mu_{1}} \mathbb{C}^{k} \otimes S_{\hbar}^{\mu_{2}} \mathbb{C}^{k} \rightarrow S_{\hbar}^{\mu_{2}} \mathbb{C}^{k} \otimes S_{\hbar}^{\mu_{1}} \mathbb{C}^{k}$ the corresponding $U_{\hbar} \mathfrak{s l}_{k}$-intertwiner, where $\sigma$ is the permutation of the tensor factors.

Proposition 6.2. The following holds on $S_{\hbar}^{\mu_{1}} \mathbb{C}^{k} \otimes S_{\hbar}^{\mu_{2}} \mathbb{C}^{k} \bigoplus S_{\hbar}^{\mu_{2}} \mathbb{C}^{k} \otimes S_{\hbar}^{\mu_{1}} \mathbb{C}^{k}$,

$$
R^{\vee} v_{i}^{\mu_{1}, \mu_{2}}=(-1)^{i} e^{\hbar\left(\left(\mu_{1}-i\right)\left(\mu_{2}-i\right)-i-\mu_{1} \mu_{2} / k\right)} v_{i}^{\mu_{2}, \mu_{1}}
$$

Proof. For any $1 \leq i \leq k-1$, let $s_{i}=(i i+1) \in \mathfrak{S}_{k}$ be the $i$ th elementary transposition and let

$$
w_{0}=(1 k)(2 k-1) \cdots\left(\left\lfloor\frac{k}{2}\right\rfloor\left\lceil\frac{k}{2}\right\rceil\right)
$$


be the longest element of $\mathfrak{S}_{k}$. Consider the following reduced expression for $w_{0}$

$$
w_{0}=s_{k-1} \cdots s_{1} s_{k-1} \cdots s_{2} \cdots \cdots s_{k-1} s_{k-2} s_{k-1}=s_{i_{1}} \cdots s_{i_{k(k-1) / 2}}
$$

and let $\beta_{j}=s_{i_{1}} \cdots s_{i_{j-1}}\left(\theta_{i_{j}}-\theta_{i_{j}+1}\right)$ be the associated enumeration of the positive roots of $\mathfrak{s l}_{k}$ so that

$$
\begin{array}{llll}
\beta_{1}=\theta_{k-1}-\theta_{k}, \quad \beta_{2}=\theta_{k-2}-\theta_{k}, & \cdots & \beta_{k-1}=\theta_{1}-\theta_{k}, \\
\beta_{k}=\theta_{k-2}-\theta_{k-1}, & \cdots & \beta_{2 k-3}=\theta_{1}-\theta_{k-1}, \\
& \ddots & \\
& & \beta_{k(k-1) / 2}=\theta_{1}-\theta_{2}
\end{array}
$$

Let $E_{\beta_{j}}, F_{\beta_{j}} \in U_{\hbar \mathfrak{s l}}, 1 \leq j \leq k(k-1) / 2$ be the corresponding quantum root vectors so that $E_{\beta_{j}}=E_{i}$ and $F_{\beta_{j}}=F_{i}$ whenever $\beta_{j}$ is the simple root $\alpha_{i}$ [Lu2, prop. 1.8]. Then, KR, LS, Ro,, $\mathrm{CP}$, thm. 8.3.9]

$$
R=\exp \left(\hbar \sum_{i=1}^{k-1} H^{i} \otimes H_{i}\right) \prod_{j=1}^{k(k-1) / 2} \exp _{q}\left(\left(q-q^{-1}\right) E_{\beta_{j}} \otimes F_{\beta_{j}}\right)
$$

where $\left\{H^{i}\right\}_{i=1}^{k-1} \subset \mathfrak{h}$ is the basis of the Cartan subalgebra of $\mathfrak{s l}_{k}$ dual to $\left\{H_{i}\right\}_{i=1}^{k-1}$ with respect to the pairing $\langle X, Y\rangle=\operatorname{tr}(X Y), q=e^{\hbar}$,

$$
\exp _{q}(x)=\sum_{n \geq 0} q^{n(n-1) / 2} \frac{x^{n}}{[n] !}
$$

and the product in (6.11) is taken so that the factor $\exp _{q}\left(\left(q-q^{-1}\right) E_{\beta_{j}} \otimes F_{\beta_{j}}\right)$ is placed to the left of $\exp _{q}\left(\left(q-q^{-1}\right) E_{\beta_{j^{\prime}}} \otimes F_{\beta_{j^{\prime}}}\right)$ whenever $j>j^{\prime}$. To compute $R v_{i}^{\mu_{1}, \mu_{2}}$, note that for any positive root $\beta \neq \theta_{1}-\theta_{2}$ and $0 \leq a \leq \mu_{1}$,

$$
E_{\beta} X_{11}^{\mu_{1}-a} X_{21}^{a}=0
$$

since, by (5.17), $\left(\mu_{1}-a, a, 0, \ldots, 0\right)+\beta$ is not a weight of $S_{\hbar}^{\mu_{1}} \mathbb{C}^{k}$. Thus, using (5.17)-(5.19)

$$
\begin{aligned}
R v_{i}^{\mu_{1}, \mu_{2}}= & \exp \left(\hbar \sum_{i=1}^{k-1} H^{i} \otimes H_{i}\right) \exp _{q}\left(\left(q-q^{-1}\right) E_{1} \otimes F_{1}\right) v_{i}^{\mu_{1}, \mu_{2}} \\
= & \sum_{\substack{0 \leq a \leq i \\
0 \leq n \leq i-a}}(-1)^{a} e^{\hbar a\left(\mu_{2}-a+1\right)}\left[\begin{array}{c}
i \\
a
\end{array}\right] e^{\hbar\left(\left(\mu_{1}-i+a+n\right)\left(\mu_{2}-a-n\right)+(i-a-n)(a+n)-\mu_{1} \mu_{2} / k\right)} \\
& \cdot \frac{e^{\hbar n(n-1) / 2}\left(e^{\hbar}-e^{-\hbar}\right)^{n}}{[n] !} \frac{[i-a] !\left[\mu_{2}-a\right] !}{[i-a-n] !\left[\mu_{2}-a-n\right] !} \\
& \cdot X_{11}^{\mu_{1}-i+a+n} X_{21}^{i-a-n} \otimes X_{12}^{\mu_{2}-a-n} X_{22}^{a+n}
\end{aligned}
$$

which, upon setting $\alpha=a+n$, yields

$$
e^{\hbar\left(\left(\mu_{1}-i\right)\left(\mu_{2}-i\right)-i-\mu_{1} \mu_{2} / k\right)} \sum_{\alpha=0}^{i}(-1)^{\alpha}\left[\begin{array}{l}
i \\
\alpha
\end{array}\right] e^{\hbar(i-\alpha)\left(\mu_{1}-i+\alpha+1\right)} S_{\alpha}^{\mu_{2}} X_{11}^{\mu_{1}-i+\alpha} X_{21}^{i-\alpha} \otimes X_{12}^{\mu_{2}-\alpha} X_{22}^{\alpha}
$$

where

$$
S_{\alpha}^{\mu}=e^{\hbar \alpha(\mu-\alpha+1)} \sum_{n=0}^{\alpha}(-1)^{n}\left[\begin{array}{l}
\alpha \\
n
\end{array}\right] \frac{[\mu-\alpha+n] !}{[\mu-\alpha] !} e^{\hbar(\alpha-n)(\mu-\alpha+n+1)+\hbar n(n-1) / 2}\left(e^{\hbar}-e^{-\hbar}\right)^{n}
$$


We claim that $S_{\alpha}^{\mu}=1$ for any $\alpha \leq \mu \in \mathbb{N}$ so that (6.7) holds. Indeed, using

$$
\left[\begin{array}{c}
\alpha \\
a
\end{array}\right]=\delta_{\alpha>a}\left[\begin{array}{c}
\alpha-1 \\
a
\end{array}\right] e^{-\hbar a}+\delta_{a>0}\left[\begin{array}{c}
\alpha-1 \\
a-1
\end{array}\right] e^{\hbar(\alpha-a)}
$$

one readily finds

$$
S_{\alpha}^{\mu}=e^{2 \hbar(\mu-\alpha+1)} S_{\alpha-1}^{\mu-1}-\left(e^{2 \hbar(\mu-\alpha+1)}-1\right) S_{\alpha-1}^{\mu}
$$

whence $S_{\alpha}^{\mu}=1$ by induction on $\alpha$ since $S_{0}^{\mu}=1$ for any $\mu \in \mathbb{N}$

6.2. Quantum Weyl group action on singular vectors. Let $E, F, H$ be the standard generators of $U_{\hbar \mathfrak{s l}}$.

Lemma 6.3. The following holds in $\mathcal{S}_{\hbar}\left(\mathcal{M}_{k, 2}^{*}\right)$,

$$
\begin{aligned}
& E v_{i}^{\mu_{1}, \mu_{2}}=\left[\mu_{2}-i\right] v_{i}^{\mu_{1}+1, \mu_{2}-1} \\
& F v_{i}^{\mu_{1}, \mu_{2}}=\left[\mu_{1}-i\right] v_{i}^{\mu_{1}-1, \mu_{2}+1} \\
& H v_{i}^{\mu_{1}, \mu_{2}}=\left(\mu_{1}-\mu_{2}\right) v_{i}^{\mu_{1}, \mu_{2}}
\end{aligned}
$$

Proof. By (5.21),

$$
\begin{aligned}
E v_{i}^{\mu_{1}, \mu_{2}}= & \sum_{a=0}^{i}(-1)^{a}\left[\begin{array}{l}
i \\
a
\end{array}\right] e^{\hbar a\left(\mu_{2}-a+1\right)}\left[\mu_{2}-a\right] e^{\hbar(i-2 a)} X_{11}^{\mu_{1}-i+a+1} X_{21}^{i-a} \otimes X_{12}^{\mu_{2}-a-1} X_{22}^{a} \\
& +\sum_{a=0}^{i}(-1)^{a}\left[\begin{array}{l}
i \\
a
\end{array}\right] e^{\hbar a\left(\mu_{2}-a+1\right)}[a] X_{11}^{\mu_{1}-i+a} X_{21}^{i-a+1} \otimes X_{12}^{\mu_{2}-a} X_{22}^{a-1} \\
= & \sum_{a=0}^{i}(-1)^{a}\left[\begin{array}{l}
i \\
a
\end{array}\right] e^{\hbar a\left(\mu_{2}-a+1\right)}\left[\mu_{2}-a\right] e^{\hbar(i-2 a)} X_{11}^{\mu_{1}-i+a+1} X_{21}^{i-a} \otimes X_{12}^{\mu_{2}-a-1} X_{22}^{a} \\
& -\sum_{a=0}^{i}(-1)^{a}\left[\begin{array}{l}
i \\
a
\end{array}\right] e^{\hbar(a+1)\left(\mu_{2}-a\right)}[i-a] X_{11}^{\mu_{1}-i+a+1} X_{21}^{i-a} \otimes X_{12}^{\mu_{2}-a-1} X_{22}^{a} \\
= & {\left[\mu_{2}-i\right] \sum_{a=0}^{i}(-1)^{a}\left[\begin{array}{l}
i \\
a
\end{array}\right] e^{\hbar a\left(\mu_{2}-a\right)} X_{11}^{\mu_{1}+1-i+a} X_{21}^{i-a} \otimes X_{12}^{\mu_{2}-1-a} X_{22}^{a} } \\
= & {\left[\mu_{2}-i\right] v_{i}^{\mu_{1}+1, \mu_{2}-1} }
\end{aligned}
$$


Similarly, by (5.22),

$$
\begin{aligned}
F v_{i}^{\mu_{1}, \mu_{2}}= & \sum_{a=0}^{i}(-1)^{a}\left[\begin{array}{l}
i \\
a
\end{array}\right] e^{\hbar a\left(\mu_{2}-a+1\right)}\left[\mu_{1}-i+a\right] X_{11}^{\mu_{1}-i+a-1} X_{21}^{i-a} \otimes X_{12}^{\mu_{2}-a+1} X_{22}^{a} \\
+ & \sum_{a=0}^{i}(-1)^{a}\left[\begin{array}{l}
i \\
a
\end{array}\right] e^{\hbar a\left(\mu_{2}-a+1\right)}[i-a] e^{-\hbar\left(\mu_{1}-\mu_{2}-i+2 a\right)} X_{11}^{\mu_{1}-i+a} X_{21}^{i-a-1} \otimes X_{12}^{\mu_{2}-a} X_{22}^{a+1} \\
= & \sum_{a=0}^{i}(-1)^{a}\left[\begin{array}{l}
i \\
a
\end{array}\right] e^{\hbar a\left(\mu_{2}-a+1\right)}\left[\mu_{1}-i+a\right] X_{11}^{\mu_{1}-i+a-1} X_{21}^{i-a} \otimes X_{12}^{\mu_{2}-a+1} X_{22}^{a} \\
& -\sum_{a=0}^{i}(-1)^{a}\left[\begin{array}{l}
i \\
a
\end{array}\right] e^{\hbar(a-1)\left(\mu_{2}-a+2\right)}[a] e^{-\hbar\left(\mu_{1}-\mu_{2}-i+2 a-2\right)} X_{11}^{\mu_{1}-i+a-1} X_{21}^{i-a} \otimes X_{12}^{\mu_{2}-a+1} X_{22}^{a} \\
= & {\left[\mu_{1}-i\right] \sum_{a=0}^{i}(-1)^{a}\left[\begin{array}{c}
i \\
a
\end{array}\right] e^{\hbar a\left(\mu_{2}-a+2\right)} X_{11}^{\mu_{1}-1-i+a} X_{21}^{i-a} \otimes X_{12}^{\mu_{2}+1-a} X_{22}^{a} } \\
= & {\left[\mu_{1}-i\right] v_{i}^{\mu_{1}-1, \mu_{2}+1} }
\end{aligned}
$$

Finally, 6.21 follows from 5.20

Let now

$$
S=\exp _{q^{-1}}\left(q^{-1} E q^{-H}\right) \exp _{q^{-1}}(-F) \exp _{q^{-1}}\left(q E q^{H}\right) q^{H(H+1) / 2}
$$

be the generator of the quantum Weyl group of $U_{\hbar \mathfrak{s l}}$ ( $[\mathrm{Lu} 3$, $\mathrm{KR}$, So, we use the form given in [Ka, Sa]) where $q=e^{\hbar}$ and the $q-$ exponential is defined by (6.12).

Proposition 6.4. The following holds in $\mathcal{S}_{\hbar}\left(\mathcal{M}_{k, 2}^{*}\right)$,

$$
S v_{i}^{\mu_{1}, \mu_{2}}=(-1)^{\mu_{1}-i} q^{\left(\mu_{1}-i\right)\left(\mu_{2}-i+1\right)} v_{i}^{\mu_{2}, \mu_{1}}
$$

Proof. Fix $\mu, i \in \mathbb{N}$ with $2 i \leq \mu$. By lemma 6.3, the vectors $u_{k}=v_{i}^{\mu-i-k, i+k}$, with $0 \leq k \leq$ $\mu-2 i$ satisfy

$$
\begin{aligned}
& E u_{k}=[k] u_{k-1} \\
& F u_{k}=[\mu-2 i-k] u_{k+1} \\
& H u_{k}=(\mu-2 i-2 k) u_{k}
\end{aligned}
$$

and therefore span the indecomposable $U_{\hbar} \mathfrak{s l}_{2}-$ module of dimension $\mu-2 i+1$. Moreover,

$$
u_{k}=\frac{[\mu-2 i-k] !}{[\mu-2 i] !} F^{k} u_{0}=\frac{[\mu-2 i-k] !}{[\mu-2 i] !} E^{\mu-2 i-k} u_{\mu-2 i}
$$

Since $\operatorname{Ad}(S) H=-H, S u_{0}$ is proportional to $u_{\mu-2 i}$ and, by (6.24) is therefore equal to

$$
(-1)^{\mu-2 i} q^{\mu-2 i} \frac{F^{\mu-2 i}}{[\mu-2 i] !} u_{0}=(-1)^{\mu} q^{\mu-2 i} u_{\mu-2 i}
$$

Next, using $\operatorname{Ad}(S) F=-q^{-H} E$ and 6.29 , we find

$$
S u_{k}=\frac{[\mu-2 i-k] !}{[\mu-2 i] !} \operatorname{Ad}(S) F^{k} S u_{0}=(-1)^{\mu-k} q^{(k+1)(\mu-2 i-k)} u_{\mu-2 i-k}
$$

Thus, setting $\mu=\mu_{1}+\mu_{2}$, so that $v_{i}^{\mu_{1}, \mu_{2}}=u_{\mu_{2}-i}$, we find

$$
S v_{i}^{\mu_{1}, \mu_{2}}=(-1)^{\mu_{1}-i} q^{\left(\mu_{1}-i\right)\left(\mu_{2}-i+1\right)} v_{i}^{\mu_{2}, \mu_{1}}
$$


as claimed

6.3. Identification of $\mathbf{R}$ and quantum Weyl group actions. Fix $1 \leq j \leq n$ and let $R_{j}^{\vee}$ be the universal $R$-matrix of $U_{\hbar} \mathfrak{g l}_{k}$ acting on the $j$ and $j+1$ tensor copies of $\mathcal{S}_{\hbar}\left(\mathcal{M}_{k, 1}^{*}\right)^{\otimes n}$. Let $S_{j}$ be the element of the quantum Weyl group of $U_{\hbar} \mathfrak{g l}_{n}$ corresponding to the simple root $\theta_{j}-\theta_{j+1}$.

Theorem 6.5. The following holds on $\mathcal{S}_{\hbar}\left(\mathcal{M}_{k, n}^{*}\right)$

$$
R_{j}^{\vee}=S_{j} \cdot e^{-\hbar\left(D_{j}^{(n)}+D_{j}^{(n)} D_{j+1}^{(n)} / k\right)} \cdot e^{i \pi D_{j}^{(n)}}
$$

Proof. Let $U_{\hbar} \mathfrak{g l}_{2}^{j} \subset U_{\hbar} \mathfrak{g l}_{n}$ be the Hopf subalgebra generated by $E_{j}^{(n)}, F_{j}^{(n)}, D_{j}^{(n)}, D_{j+1}^{(n)}$. By (5.20)-(5.22),$U_{\hbar} \mathfrak{g l}_{2}^{j}$ only acts upon the variables $X_{i j}, X_{i j+1}, 1 \leq i \leq k$. Thus, $U_{\hbar \mathfrak{g l}} \mathfrak{l}_{2}^{j}$ and therefore $S_{j}$ only act on the $j$ and $j+1$ tensor copies $\mathcal{S}_{\hbar}\left(\mathcal{M}_{k, 1}^{*}\right)_{j}, \mathcal{S}_{\hbar}\left(\mathcal{M}_{k, 1}^{*}\right)_{j+1}$ of $\mathcal{S}_{\hbar}\left(\mathcal{M}_{k, 1}^{*}\right)^{\otimes n} \cong$ $\mathcal{S}_{\hbar}\left(\mathcal{M}_{k, n}^{*}\right)$. Since this is also the case of $R_{j}^{\vee}$, the proof of (6.33) reduces to a computation on the $U_{\hbar} \mathfrak{g l}_{k} \otimes U_{\hbar} \mathfrak{g l}_{2}^{j}$-module $\mathcal{S}_{\hbar}\left(\mathcal{M}_{k, 1}^{*}\right)_{j} \otimes \mathcal{S}_{\hbar}\left(\mathcal{M}_{k, 1}^{*}\right)_{j+1} \cong \mathcal{S}_{\hbar}\left(\mathcal{M}_{k, 2}^{*}\right)$. Both sides of (6.33) clearly commute with $U_{\hbar} \mathfrak{g l}_{k}$, so it is suffices to check their equality on the singular vectors of

$$
\mathcal{S}_{\hbar}\left(\mathcal{M}_{k, 1}^{*}\right)_{j} \otimes \mathcal{S}_{\hbar}\left(\mathcal{M}_{k, 1}^{*}\right)_{j+1}=\bigoplus_{\mu_{1}, \mu_{2} \in \mathbb{N}} S_{\hbar}^{\mu_{1}} \mathbb{C}^{k} \otimes S_{\hbar}^{\mu_{2}} \mathbb{C}^{k}
$$

i.e., on the vectors $v_{i}^{\mu_{1}, \mu_{2}} \in S_{\hbar}^{\mu_{1}} \mathbb{C}^{k} \otimes S_{\hbar}^{\mu_{2}} \mathbb{C}^{k}$ of lemma 6.1. By propositions 6.2 and 6.4,

$$
R_{j}^{\vee} v_{i}^{\mu_{1}, \mu_{2}}=S_{j} \cdot e^{-\hbar\left(\mu_{1}+\mu_{1} \mu_{2} / k\right)}(-1)^{\mu_{1}} v_{i}^{\mu_{1}, \mu_{2}}
$$

whence 6.33 since, for any $1 \leq l \leq n, D_{l}^{(n)}$ gives the $\mathbb{N}$-grading on $\mathcal{S}_{\hbar}\left(\mathcal{M}_{k, 1}^{*}\right)_{l}$

REMARK. The coincidence of the two representations of $B_{n}$ studied above was also noted by Baumann $[\mathrm{Ba}$, prop. 12] in the special case when both actions are restricted to the subspace $S_{\hbar}^{\mu_{1}} \mathbb{C}^{k} \otimes \cdots \otimes S_{\hbar}^{\mu_{n}} \mathbb{C}^{k}$ of $\mathcal{S}_{\hbar}\left(\mathcal{M}_{k, n}^{*}\right)$ where $\mu_{1}=\cdots=\mu_{n}=1$

\section{Monodromic realisation of quantum Weyl group operators}

The following is the main result of this paper. It was conjectured for any simple Lie algebra $\mathfrak{g}$ by De Concini around 1995 (unpublished) and, independently, in [T]. We prove it here for $\mathfrak{g}=\mathfrak{s l}(\mathbb{C})$.

Theorem 7.1. Let $\mathfrak{g}=\mathfrak{s l}_{n}(\mathbb{C}), V$ a finite-dimensional $\mathfrak{g}$-module, $\mu$ a weight of $V$ and

$$
V^{\mu}=\bigoplus_{\nu \in W \mu} V[\nu]
$$

the direct sum of the weight spaces of $V$ corresponding to the Weyl group orbit of $\mu$. Let $\pi_{\kappa}^{h}: B_{\mathfrak{g}} \rightarrow G L\left(V^{\mu} \llbracket h \rrbracket\right)$ be the monodromy representation of the connection

$$
d-h \sum_{\alpha>0} \frac{d \alpha}{\alpha} \pi_{V}\left(\kappa_{\alpha}\right)
$$

obtained by regarding $h$ as a formal variable. Let $\pi_{W}: B_{\mathfrak{g}} \rightarrow G L\left(V^{\mu} \llbracket h \rrbracket\right)$ be the quantum

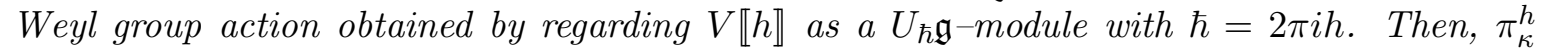
and $\pi_{W}$ are equivalent. 
Proof. We may assume that $V$ is irreducible with highest weight $\lambda=\left(\lambda_{1}, \ldots, \lambda_{n}\right)$ where $\lambda_{i} \in \mathbb{N}$. Regard $V$ as a $\mathfrak{g l}_{n}$-module by letting $1^{(n)}=\sum_{j=1}^{n} E_{j j}^{(n)}$ act as multiplication by $|\lambda|=\sum_{i=1}^{n} \lambda_{i}$ and fix some $k \geq n$. By lemma 3.4, $V[\nu]$ is isomorphic to the space $M_{\lambda}^{\nu}$ of singular vectors of weight $\lambda$ for the diagonal action of $\mathfrak{g l}_{k}$ on

$$
\mathcal{S}^{\nu} \mathbb{C}^{k}=\mathcal{S}^{\nu_{1}} \mathbb{C}^{k} \otimes \cdots \otimes \mathcal{S}^{\nu_{n}} \mathbb{C}^{k} \subset \mathcal{S}\left(\mathcal{M}_{k, n}^{*}\right)
$$

and, by corollary 3.6, the monodromy representation of the Knizhnik-Zamolodchikov connection (3.17) on $\bigoplus_{\nu \in \mathfrak{S}_{k}} M_{\lambda}^{\nu}$ and $\pi_{\kappa}^{h}$ are related by

$$
\pi_{\mathrm{KZ}}^{\bar{h}}\left(T_{j}\right)=\pi_{\kappa}^{h}\left(T_{j}\right) e^{-\pi i h\left(E_{j j}^{(n)}+E_{j+1 j+1}^{(n)}+2 E_{j j}^{(n)} E_{j+1 j+1}^{(n)} / k\right)} e^{i \pi E_{j j}^{(n)}}
$$

where $\bar{h}=2 h$.

We shall now use the Kohno-Drinfeld theorem to relate $\pi_{\mathrm{Kz}}^{\bar{h}}$ to the $R$-matrix representation of $B_{n}$ corresponding to the action of $U_{\hbar} \mathfrak{g l}_{k}$ on $\mathcal{S}^{\nu} \mathbb{C}^{k} \llbracket \hbar \rrbracket$. Let for this purpose $\phi: U_{\hbar} \mathfrak{g l}_{k} \rightarrow U \mathfrak{g l}_{k} \llbracket \hbar \rrbracket$ be an algebra isomorphism whose reduction mod $\hbar$ is the identity and which acts as the identity on the Cartan subalgebras i.e., $\phi\left(D_{i}^{(k)}\right)=E_{i i}^{(k)}, 1 \leq i \leq k$ [Dr2, prop. 4.3]. Then, $U_{\hbar \mathfrak{g l}_{k}}$ act on each $\mathcal{S}^{\nu_{j}} \mathbb{C}^{k} \llbracket \hbar \rrbracket$ via $\phi$ and on

$$
\mathcal{S}^{\nu_{1}} \mathbb{C}^{k} \llbracket \hbar \rrbracket \otimes \cdots \otimes \mathcal{S}^{\nu_{n}} \mathbb{C}^{k} \llbracket \hbar \rrbracket=\mathcal{S}^{\nu_{1}} \mathbb{C}^{k} \otimes \cdots \otimes \mathcal{S}^{\nu_{n}} \mathbb{C}^{k} \llbracket \hbar \rrbracket=\mathcal{S}^{\nu} \mathbb{C}^{k} \llbracket \hbar \rrbracket
$$

via the $n$-fold coproduct $\Delta^{(n)}: U_{\hbar} \mathfrak{g l}_{k} \rightarrow U_{\hbar} \mathfrak{g l}_{k}^{\otimes n}$ recursively defined by

$$
\begin{aligned}
\Delta^{(1)} & =\mathrm{id} \\
\Delta^{(a+1)} & =\Delta \otimes \mathrm{id}^{\otimes(a-1)} \circ \Delta^{(a)}, \quad a \geq 1
\end{aligned}
$$

where $\Delta=\Delta^{(2)}$ is given by 4.9$)-(4.11)$.

Let $\Delta_{0}$ be the standard, cocommutative coproduct on $U \mathfrak{g l}_{k}$ so that $U \mathfrak{g l}_{k}$ acts on $\mathcal{S}^{\nu} \mathbb{C}^{k}$ via $\Delta_{0}^{(n)}: U \mathfrak{g l}_{k} \rightarrow U \mathfrak{g l}_{k}^{\otimes(n)}$ defined as in (7.6)-(7.7) with $\Delta$ replaced by $\Delta_{0}$. Since $\Delta=\Delta_{0}+o(\hbar)$ and $H^{1}\left(\mathfrak{s l}_{k}, \mathfrak{s l}_{k} \oplus \mathfrak{s l}_{k}\right)=0$, there exists a twist $F=1 \otimes 1+o(\hbar) \in U \mathfrak{g l}_{k}^{\otimes(2)} \llbracket \hbar \rrbracket$ such that, for any $x \in U_{\hbar} \mathfrak{g l}_{k}$,

$$
\phi \otimes \phi \circ \Delta(x)=F \Delta_{0}(\phi(x)) F^{-1}
$$

It follows that the actions of $U_{\hbar} \mathfrak{g l}_{k}$ and $U \mathfrak{g l}_{k} \llbracket \hbar \rrbracket$ on $\mathcal{S}^{\nu} \mathbb{C}^{k}$ are related by

$$
\phi^{\otimes(n)} \circ \Delta^{(n)}(x)=F^{(n)} \Delta_{0}^{(n)}(\phi(x)) F^{(n)^{-1}}, \quad x \in U_{\hbar} \mathfrak{g l}_{k}
$$

where $F^{(n)} \in U \mathfrak{g l}_{k}^{\otimes n} \llbracket \hbar \rrbracket$ is recursively defined by

$$
\begin{aligned}
F^{(1)} & =1 \\
F^{(a+1)} & =F \otimes 1^{\otimes(a-1)} \cdot \Delta_{0} \otimes \mathrm{id}^{\otimes(a-1)}\left(F^{(a)}\right), \quad a \geq 1
\end{aligned}
$$

Let now $M_{\lambda}^{\nu} \subset \mathcal{S}^{\nu} \mathbb{C}^{k}$ and $M_{\hbar, \lambda}^{\nu} \subset \mathcal{S}^{\nu} \mathbb{C}^{k} \llbracket \hbar \rrbracket$ be the subspaces of vectors of highest weight $\lambda$ for the actions of $\mathfrak{g l}_{k}$ and $U_{\hbar} \mathfrak{g l}_{k}$ respectively. We shall need the following

Lemma 7.2. $F^{(n)} M_{\lambda}^{\nu \llbracket \hbar \rrbracket}=M_{\hbar, \lambda}^{\nu}$.

Proof. Let $\mathcal{S}^{\nu} \mathbb{C}^{k}(\lambda) \subset \mathcal{S}^{\nu} \mathbb{C}^{k}$ and $\mathcal{S}^{\nu} \mathbb{C}^{k} \llbracket \hbar \rrbracket(\lambda) \subset \mathcal{S}^{\nu} \mathbb{C}^{k} \llbracket \hbar \rrbracket$ be the isotypical components of types $V_{\lambda}$ and $V_{\lambda} \llbracket \hbar \rrbracket$ for $\mathfrak{g l}_{k}$ and $U_{\hbar} \mathfrak{g l}_{k}$ respectively. The subspace $F^{(n)} \mathcal{S}^{\nu} \mathbb{C}^{k}(\lambda) \llbracket \hbar \rrbracket$ is invariant under $U_{\hbar \mathfrak{g l}_{k}}$ by $(7.9)$ and its reduction $\bmod \hbar$ is equal to $\mathcal{S}^{\nu} \mathbb{C}^{k}(\lambda)$ since $F^{(n)}=1^{\otimes(n)}+$ $o(\hbar)$. Thus, by proposition 4.1, $F^{(n)} \mathcal{S}^{\nu} \mathbb{C}^{k}(\lambda) \llbracket \hbar \rrbracket$ is isomorphic to $\mathcal{S}^{\nu} \mathbb{C}^{k}(\lambda) \llbracket \hbar \rrbracket$ and therefore contained in $\mathcal{S}^{\nu} \mathbb{C}^{k} \llbracket \hbar \rrbracket(\lambda)$. Since this holds for any $\lambda$, the inclusion is an equality. Noting 
now that $F^{(n)}$ is equivariant for the action of the Cartan subalgebras of $\mathfrak{g l}_{k}$ and $U_{\hbar} \mathfrak{g l}_{k}$ since $\Delta_{0} \circ \phi(h)=\phi \otimes \phi \circ \Delta(h)$ for any $h \in \mathfrak{h}$, we get that

$$
\begin{aligned}
F^{(n)} M_{\lambda}^{\nu \llbracket \hbar \rrbracket} & =F^{(n)}\left(\mathcal{S}^{\nu} \mathbb{C}^{k}(\lambda)[\lambda] \llbracket \hbar \rrbracket\right) \\
& =F^{(n)}\left(\mathcal{S}^{\nu} \mathbb{C}^{k}(\lambda) \llbracket \hbar \rrbracket\right)[\lambda] \\
& =\mathcal{S}^{\nu} \mathbb{C}^{k} \llbracket \hbar \rrbracket(\lambda)[\lambda] \\
& =M_{\hbar, \lambda}^{\nu}
\end{aligned}
$$

as claimed

Summarising, we have an action of $B_{n}$ on $\bigoplus_{\nu \in \mathfrak{S}_{n} \mu} M_{\lambda}^{\nu}$ via the monodromy of the KnizhnikZamolodchikov connection and an action of $B_{n}$ on $\bigoplus_{\nu \in \mathfrak{S}_{n} \mu} M_{\hbar, \lambda}^{\nu}$ via the $R$-matrix representation of $U_{\hbar \mathfrak{s l}}$. Drinfeld's theorem [Dr3, Dr4, Dr5] asserts that the twist $F$ may be chosen so that

$$
F^{(n)}: \bigoplus_{\nu \in \mathfrak{S}_{n} \mu} M_{\lambda}^{\nu} \llbracket \hbar \rrbracket \longrightarrow \bigoplus_{\nu \in \mathfrak{S}_{n} \mu} M_{\hbar, \lambda}^{\nu}
$$

is $B_{n}$-equivariant so that, for any $1 \leq j \leq n-1$,

$$
\pi_{\mathrm{KZ}}^{\bar{h}}\left(T_{j}\right)=F^{(n)^{-1}} R_{j}^{\vee} F^{(n)}
$$

where $R_{j}^{\vee}$ is the universal $R$-matrix for $U_{\hbar \mathfrak{s l}}$ acting on the $j$ and $j+1$ copies of $\bigoplus_{\nu \in \mathfrak{S}_{n} \mu} \mathcal{S}^{\nu} \mathbb{C}^{k} \llbracket \hbar \rrbracket$ and $\hbar=\pi i \bar{h}$.

Let now $\mathcal{S}_{\hbar}\left(\mathcal{M}_{k, n}^{*}\right) \cong\left(\mathcal{S}_{\hbar}\left(\mathcal{M}_{k, 1}^{*}\right)\right)^{\otimes n}$ be the algebra of functions on quantum $k \times n$ matrix space defined in section 5 and, for any $\nu \in \mathbb{N}^{n}$, let

$$
\mathcal{S}_{\hbar}^{\nu} \mathbb{C}^{k}=\mathcal{S}_{\hbar}^{\nu_{1}} \mathbb{C}^{k} \otimes \cdots \otimes \mathcal{S}_{\hbar}^{\nu_{n}} \mathbb{C}^{k}
$$

be the space of polynomials which are homogeneous of degree $\nu_{j}$ in the variables $X_{1 j}, \ldots, X_{k j}$, for any $1 \leq j \leq n$. By (5.17)-(5.19), $\mathcal{S}_{\hbar}^{\nu} \mathbb{C}^{k} / \hbar \mathcal{S}_{\hbar}^{\nu} \mathbb{C}^{k}$ is the $\mathfrak{g l}_{k}-$ module $\mathcal{S}^{\nu} \mathbb{C}^{k}$ so that, by proposition 4.1, we may identify $\mathcal{S}_{\hbar}^{\nu} \mathbb{C}^{k}$ with $\mathcal{S}^{\nu} \mathbb{C}^{k} \llbracket \hbar \rrbracket$ as $U_{\hbar} \mathfrak{g l}_{k}-$ modules.

By theorem 5.5 and the fact that the $\mathbb{N}$-grading on the $j$ th tensor factor of $\mathcal{S}_{\hbar}\left(\mathcal{M}_{k, n}^{*}\right) \cong$ $\left(\mathcal{S}_{\hbar}\left(\mathcal{M}_{1, k}^{*}\right)\right)^{\otimes n}$ is given by the action of $D_{j}^{(n)}$, the space $M_{\hbar, \lambda}^{\nu}$ is isomorphic to the subspace $V_{\lambda}^{(n)}[\nu] \llbracket \hbar \rrbracket \subset V_{\lambda}^{(n)} \llbracket \hbar \rrbracket$ of weight $\nu$. Using now theorem 6.5, we find

$$
\begin{aligned}
\pi_{\kappa}^{h}\left(T_{j}\right) & =F^{(n)^{-1}} \cdot S_{j} \cdot e^{-\hbar\left(D_{j}^{(n)}+D_{j}^{(n)} D_{j+1}^{(n)} / k\right)} \cdot e^{i \pi D_{j}^{(n)}} \cdot F^{(n)} \\
& \cdot e^{\pi i h\left(E_{j}^{(n)}+E_{j+1}^{(n)}+2 E_{j}^{(n)} E_{j+1}^{(n)} / k\right)} \cdot e^{i \pi E_{j}^{(n)}}
\end{aligned}
$$

where $S_{j}=\pi_{W}\left(T_{j}\right)$ is the quantum Weyl group element of $U_{\hbar} \mathfrak{g l}_{n}$ corresponding to the simple root $\alpha_{j}=\theta_{j}-\theta_{j+1}$. Since for any $l, E_{l l}^{(n)}=D_{l}^{(n)}$ on $V^{\mu} \cong \bigoplus_{\nu \in \mathfrak{S}_{k}} M_{\lambda}^{\nu}$ we get

$$
\begin{aligned}
\pi_{\kappa}^{h}\left(T_{j}\right) & =F^{(n)^{-1}} S_{j} e^{-\hbar / 2\left(D_{j}^{(n)}-D_{j+1}^{(n)}\right)} F^{(n)} \\
& =F^{(n)^{-1}} S_{j} e^{-\hbar H_{j}^{(n)} / 2} F^{(n)} \\
& =F^{(n)^{-1}} e^{\hbar \rho^{(n)} / 2} S_{j} e^{-\hbar \rho^{(n)} / 2} F^{(n)}
\end{aligned}
$$

where $\rho^{(n)}=\frac{1}{2} \sum_{j=1}(n-2 j+1) D_{j}^{(n)}$ is the half-sum of the positive (co)roots of $\mathfrak{s l}_{n}$ 
REMARK. Theorem 7.1 is proved in [TL] for the following pairs $(\mathfrak{g}, V)$ where $\mathfrak{g}$ is a simple Lie algebra and $V$ an irreducible, finite-dimensional representation

1. $\mathfrak{g}=\mathfrak{s l}_{2}$ and $V$ is any irreducible representation.

2. $\mathfrak{g}=\mathfrak{s l}_{n}$ and $V$ is a fundamental representation.

3. $\mathfrak{g}=\mathfrak{s o}_{n}$ and $V$ is the vector or a spin representation.

4. $\mathfrak{g}=\mathfrak{s p}_{n}$ and $V$ is the defining vector representation.

5. $\mathfrak{g}=\mathfrak{e}_{6}, \mathfrak{e}_{7}$ and $V$ is a minuscule representation.

6 . $\mathfrak{g}=\mathfrak{g}_{2}$ and $V$ is the 7 -dimensional representation.

7. $\mathfrak{g}$ is any simple Lie algebra and $V \cong \mathfrak{g}$ its adjoint representation.

\section{REFERENCES}

[Ba] P. Baumann, The q-Weyl Group of a q-Schur Algebra, preprint, 1999.

[Br] E. Brieskorn, Die Fundamentalgruppe des Raumes der Regulären Orbits einer Endlichen Komplexen Spiegelungsgruppe, Invent. Math. 12 (1971), 57-61.

[CP] V. Chari, A. Pressley, A Guide to Quantum Groups. Cambridge University Press, Cambridge, 1994.

[Dr1] V. G. Drinfeld, Quantum Groups, Proceedings of the International Congress of Mathematicians, Berkeley 1986, 798-820.

[Dr2] V. G. Drinfeld, On Almost Cocommutative Hopf Algebras, Leningrad Math. J. 1 (1990), 321-342.

[Dr3] V. G. Drinfeld, Quasi-Hopf Algebras, Leningrad Math. J. 1 (1990), 1419-57.

[Dr4] V. G. Drinfeld, On Quasitriangular Quasi-Hopf Algebras and on a Group that is closely connected with $\operatorname{Gal}(\bar{Q} / Q)$, Leningrad Math. J. 2 (1991), 829-860.

[Dr5] V. G. Drinfeld, On the Structure of Quasitriangular Quasi-Hopf Algebras, Functional Anal. Appl. 26 (1992), 63-65.

[FMTV] G. Felder, Y. Markov, V. Tarasov, A. Varchenko, Differential Equations Compatible with KZ Equations, Math. Phys. Anal. Geom. 3 (2000), 139-177.

[Ga] F. Galdi, Ph.D. dissertation, Università di Roma II "Tor Vergata", 1995.

[GS] V. Guillemin, S. Sternberg, The Gel'fand-Cetlin System and Quantization of the Complex Flag Manifolds, J. Funct. Anal. 52 (1983), 106-128.

[HK] J.-C. Hausmann, A. Knutson, Polygon Spaces and Grassmannians. Enseign. Math. 43 (1997), $173-198$.

[Ho] R. Howe, Remarks on Classical Invariant Theory, Trans. Amer. Math. Soc. 313 (1989), 539-570.

[Ji1] M. Jimbo, A q-Difference Analogue of $U(\mathfrak{g})$ and the Yang-Baxter Equation. Lett. Math. Phys. 10 (1985), 63-69.

[Ji2] M. Jimbo, A q-Analogue of $U \mathfrak{g l}(N+1)$, Hecke Algebra, and the Yang-Baxter Equation, Lett. Math. Phys. 11 (1986), 247-252.

[KM] M. Kapovich, J. Millson, The Symplectic Geometry of Polygons in Euclidean Space, J. Differential Geom. 44 (1996), 479-513.

[Ka] M. Kashiwara, Private Note on Finite-Dimensional Representations of Quantized Affine Algebras, unpublished notes.

[KR] A. N. Kirillov, N. Reshetikhin, q-Weyl Group and a Multiplicative Formula for Universal R-Matrices, Comm. Math. Phys. 134 (1990), 421-431.

[Kn] A. Knutson, personal communication to J. Millson, May 1999.

[Ko1] T. Kohno, Quantized Enveloping Algebras and Monodromy of Braid Groups, preprint, 1988.

[Ko2] T. Kohno, Integrable Connections Related to Manin and Schechtman's Higher Braid Groups, Illinois J. Math. 34 (1990), 476-484.

[LS] S. Z. Levendorskii, Y. S. Soibelman, Some applications of the Quantum Weyl Groups, J. Geom. Phys. 7 (1990), 241-254.

[Lu1] G. Lusztig, On Quantum Groups, J. Algebra 131 (1990), 466-475.

[Lu2] G. Lusztig, Finite-Dimensional Hopf Algebras Arising from Quantized Universal Enveloping Algebra. J. Amer. Math. Soc. 3 (1990), 257-296.

[Lu3] G. Lusztig, Introduction to Quantum Groups. Progress in Mathematics, 110. Birkhäuser Boston, 1993.

[Mc] I. G. Macdonald, Symmetric Functions and Hall Polynomials. 2nd edition, Oxford University Press, 1995.

[MTL] J. Millson, V. Toledano Laredo, Casimir Operators and Monodromy Representations of Generalised Braid Groups, in preparation.

[PW] B. Parshall, J.-P. Wang, Quantum Linear Groups. Mem. Amer. Math. Soc. 89 (1991), n. 439. 
[Ro] M. Rosso, An analogue of P.B.W. Theorem and the Universal R-Matrix for $U_{h} \operatorname{sl}(N+1)$. Comm. Math. Phys. 124 (1989), 307-318.

[Sa] Y. Saito, PBW Basis of Quantized Universal Enveloping Algebras, Publ. Res. Inst. Math. Sci. 30 (1994), 209-232.

[So] Y.S. Soibelman, Algebra of Functions on a Compact Quantum Group and its Representations, Leningrad Math. J. 2 (1991), 161-178.

[TV] V. Tarasov, A. Varchenko, Difference Equations Compatible with Trigonometric KZ Differential Equations, Internat. Math. Res. Notices 2000, 801-829.

[Ti] J. Tits, Normalisateurs de Tores. I. Groupes de Coxeter Etendus, J. Algebra 4 (1966), 96-116.

[TL] V. Toledano Laredo, Monodromy Representations of Generalised Braid Groups and Quantum Weyl Groups, in preparation.

[Zh] D. P. Zhelobenko, Compact Lie Groups and their Representations., Translations of Mathematical Monographs, Vol. 40. American Mathematical Society, 1973.

MSRI

1000 Centennial Drive

Berkeley, CA 94720-5070

TOLEDANO@MSRI.ORG

PERMANENT ADDRESS :

Institut De Mathematiques De Jussieu

UMR 7586, CASE 191

Universite Pierre et Marie Curie

4, Place Jussieu

F-75252 PARIS CEDEX 05

TOLEDANO@MATH.JUSSIEU.FR 